\title{
The System Barium Oxide-Boric Oxide-Silica
}

\author{
Ernest M. Levin and George M. Ugrinic
}

\begin{abstract}
A phase equilibrium diagram for the system $\mathrm{BaO}-\mathrm{B}_{2} \mathrm{O}_{3}-\mathrm{SiO}_{2}$ has been constructed from data, obtained essentially by the quenching method, on 178 ternary compositions. One new compound, $3 \mathrm{Ba} \cdot \mathrm{O}_{3} \mathrm{~B}_{2} \mathrm{O}_{3} \cdot 2 \mathrm{SiO}_{2}$, melting at $1,009^{\circ} \mathrm{C}$ and possessing an extremely flat primary field appears, and its optical properties and X-ray diffraction data are given. The $\mathrm{BaO} \cdot 4 \mathrm{~B}_{2} \mathrm{O}_{3}$ and $\mathrm{SiO}_{2}$ primary phase areas include almost 70 percent of the diagram, and over these areas exists a large region of two-liquid immiscibility. Limited data indicates that the immiscibility gap decreases with increasing temperature. The solid solution region between $2 \mathrm{BaO} \cdot 3 \mathrm{SiO}_{2}$ and $\mathrm{BaO} \cdot 2 \mathrm{SiO}_{2}$ extends into the interior of the diagram and is complex in nature. An isofract diagram for the quenched glasses is shown. For precise temperature control of a quenching furnace, a self-adjusting, bridge-type controller is described briefly.
\end{abstract}

\section{Introduction}

The system barium oxide-boric oxide-silica is of fundamental importance in the ceramic field, and the three end members comprise the major constituents of the large class of barium crown glasses. Other important constituents maybe aluminum- or zincoxide in the dense barium crown glasses, lead in the barium flints, and alkalis in the light barium crowns or flints [1]. ${ }^{1}$ Barium oxide surpasses nearly all other oxides in imparting both high index and low dispersion to glass. Many of the well-corrected, present-day optical systems, such as are found in photographic and projectional equipment, have resulted largely from the use of high barium-oxidecontent glasses, which have a favorable relationship between index and dispersion.

The Glass Section of the National Bureau of Standards has been engaged in an extensive study of the refractivity, dispersion, and density in the glassforming region of the barium-borosilicate system [2]. The phase equilibrium diagram for the system is of paramount importance in the interpretation of the data as regards the structure of these glasses. The high silica-boric oxide portion of the system, also, has possible application to the development of improved enamels and ceramic glazes. Notwithstanding the fundamental and practical importance of the ternary diagram, however, no systematic study of the phase equilibrium relationships has been reported.

The ternary system is of additional interest from several theoretical considerations. It contains one binary- system $\left(\mathrm{BaO}-\mathrm{B}_{2} \mathrm{O}_{3}\right)$ with an immiscibility gap and another $\left(\mathrm{BaO}-\mathrm{SiO}_{2}\right)$ with a portion of the liquidus curve approaching that of two-liquid immiscibility. In the $\mathrm{BaO}-\mathrm{SiO}_{2}$ system, $\mathrm{BaO} \cdot 2 \mathrm{SiO}_{2}$ and $2 \mathrm{BaO} \cdot 3 \mathrm{SiO}_{2}$ show unusual behavior for refractory oxide systems, in that they form a complete isomorphous solid solution series without a maximum or a minimum. As a secondary objective, it was hoped that investigation of the ternary system might provide additional information on these problems.

${ }^{1}$ Figures in brackets indicate the literature references at the end of this paper.

\section{Experimental Procedure}

\subsection{Raw Materials}

The starting materials were silica gel $\left(\mathrm{SiO}_{2} \cdot n \mathrm{H}_{2} \mathrm{O}\right)$, boric acid $\left(\mathrm{H}_{3} \mathrm{BO}_{3}\right)$, and either barium nitrate $\left(\mathrm{Ba}\left(\mathrm{NO}_{3}\right)_{2}\right)$ or barium carbonate $\left(\mathrm{BaCO}_{3}\right)$. The silica gel, 99.99 percent pure on an ignited weight basis, was specially prepared by an acid digestion process in the Chemistry Division of the National Bureau of Standards. The remaining starting materials were of reagent quality, meeting ACS specifications.

\subsection{Preparation and Analysis of Mixtures}

Because of the volatility of boric acid and the extreme hygroscopicity of boric oxıde [3, 4], it was found advantageous in preparing ternary mixtures to add the boric oxide already combined with the baria. For this purpose a stock quantity of each of the four barium borates, $3 \mathrm{BaO} \cdot \mathrm{B}_{2} \mathrm{O}_{3}, \mathrm{BaO} \cdot \mathrm{B}_{2} \mathrm{O}_{3}$, $\mathrm{BaO} \cdot 2 \mathrm{~B}_{2} \mathrm{O}_{3}, \mathrm{BaO} \cdot 4 \mathrm{~B}_{2} \mathrm{O}_{3}$, was prepared by mixing, grinding, sintering or fusing the appropriate amounts of boric acid and barium nitrate or carbonate. The product was ground to pass a No. 200 mesh screen and heated again. The process of grinding and reheating was repeated 2 or 3 times, until a homogeneous product, by petrographic examination, was obtained. Barium carbonate was used as the source of $\mathrm{BaO}$ for preparing $3 \mathrm{BaO} \cdot \mathrm{B}_{2} \mathrm{O}_{3}$; for the other borates, barium nitrate was used. The barium borates thus prepared were analyzed [4] for $\mathrm{BaO}$ and $\mathrm{B}_{2} \mathrm{O}_{3}$. Previous experience had indicated the approximate excess of boric acid required to focmulate each compound, and in no case did the actual chemical composition vary from the theoretical compound composition by more than 0.5 percent. No attempt was made to adjust compositions to theoretical values, and the analyzed values were applied in subsequent calculations.

Stock quantities of the 4 barium silicates, $2 \mathrm{BaO} \cdot \mathrm{SiO}_{2}, \mathrm{BaO} \cdot \mathrm{SiO}_{2}, 2 \mathrm{BaO} \cdot 3 \mathrm{SiO}$, and $\mathrm{BaO} \cdot 2 \mathrm{SiO}_{2}$, 
TABLE 1. Agreement between some formulated and analyzed compositions

\begin{tabular}{|c|c|c|c|c|c|c|c|}
\hline \multirow{3}{*}{ Composition number } & \multirow{3}{*}{ Starting materials } & \multicolumn{6}{|c|}{ Composition } \\
\hline & & \multicolumn{3}{|c|}{ Formulated a } & \multicolumn{3}{|c|}{ Analyzed b } \\
\hline & & $\mathrm{BaO}$ & $\mathrm{B}_{2} \mathrm{O}_{3}$ & $\mathrm{SiO}_{2}$ & $\mathrm{BaO}$ & $\mathrm{B}_{2} \mathrm{O}_{3}$ & $\mathrm{SiO}_{2}$ \\
\hline $\begin{array}{l}\mathrm{C}-2 \mathrm{BaO} \cdot \mathrm{SiO}_{2} \mathrm{C} \\
\mathrm{E}-\mathrm{BaO} \cdot 2 \mathrm{SiO}_{2} \mathrm{C} \\
24 \\
\mathrm{~J}-3 \mathrm{BaO} \cdot 3 \mathrm{~B}_{2} \mathrm{O}_{3} \cdot 2 \mathrm{SiO}_{2} \mathrm{c}\end{array}$ & $\begin{array}{l}\mathrm{BaCO}_{3}, \mathrm{SiO}_{2} \\
\mathrm{BaCO}_{3}, \mathrm{SiO}_{2} \\
\mathrm{BaO} \cdot 2 \mathrm{~B}_{2} \mathrm{O}_{3}, 2 \mathrm{BaO} \cdot 3 \mathrm{SiO}_{2} \\
\mathrm{BaO} \cdot 4 \mathrm{~B}_{2} \mathrm{O}_{3}, \mathrm{BaO} \cdot 2 \mathrm{SiO}_{2} \\
\text { d } \mathrm{BaCO} \mathrm{CO}_{3}, \mathrm{SiO}_{2}, \mathrm{H}_{3} \mathrm{BO}_{3}\end{array}$ & $\begin{array}{c}\% \\
83.63 \\
56.08 \\
55.24 \\
47.69 \\
58.30\end{array}$ & $\begin{array}{c}\% \\
35.60 \\
25.92 \\
26.48\end{array}$ & \begin{tabular}{r}
\multicolumn{1}{c}{$\%$} \\
16.37 \\
43.92 \\
9.26 \\
26.39 \\
15.22
\end{tabular} & $\begin{array}{c}\% \\
83.53 \\
55.94 \\
55.11 \\
47.78 \\
58.48\end{array}$ & $\begin{array}{c}\% \\
35.51 \\
25.98 \\
26.24\end{array}$ & $\begin{array}{r}\% \\
16.47 \\
44.06 \\
9.38 \\
26.24 \\
15.28\end{array}$ \\
\hline
\end{tabular}

a From composition and amounts of starting materials.

b By analytical determination.

c Stock supply.

c Stock supply.

and of 1 ternary compound, $3 \mathrm{BaO} \cdot 3 \mathrm{~B}_{2} \mathrm{O}_{3} \cdot 2 \mathrm{SiO}_{2}$, were prepared in a similar manner. Barium carbonate exclusively was used as the source of $\mathrm{BaO}$ in preparing the barium silicates.

Most of the ternary compositions were prepared to lie on joins connecting the compositions of barium borates, barium silicates, and the one ternary compound $3 \mathrm{BaO} \cdot 3 \mathrm{~B}_{2} \mathrm{O}_{3} \cdot 2 \mathrm{SiO}_{2}$. In making up such series, the amounts of conjugate stock compounds required to yield $3-\mathrm{g}$ mixtures were calculated, by applying the well-known lever principle. The calculated amounts, weighed into dry bottles, were shaken thoroughly with a mechanical mixer for a minimum of $1 \mathrm{hr}$, ground with a mechanical grinder for $1 \mathrm{hr}$, and then heated for several hours at some temperature between the solidus and the liquidus. The products were ground to pass a No. 200 mesh sieve and were resintered at a slightly higher temperature, but still below the liquidus. Once again, the products were ground and passed through a No. 200 mesh sieve. This treatment, in most cases, gave a fairly uniform product, as examined with a polarizing microscope. In a few instances, it was necessary to heat the material a third time. All heating was done in platinum crucibles by using an electrically heated furnace. Analysis of representative mixtures prepared as described showed satisfactory agreement between actual and theoretical compositions. Table 1 gives this agreement for several compounds and mixtures. The few compositions not on tie lines were made from appropriate quantities of either 2 already prepared mixtures or 3 stock compounds.

Analysis of ternary compositions consisted of determinations for ignition loss, silica, and baria; boric oxide was obtained by difference. Half-gram samples were decomposed by $1: 1 \mathrm{HCl}$, evaporated almost to dryness, and the moist residue evaporated three times with $10-\mathrm{ml}$ portions of methyl alcohol to remove the boric oxide. Silica was determined by double dehydration in the usual way; baria was precipitated and weighed as $\mathrm{BaSO}_{4}$.

Figure 1 shows the 178 compositions investigated, together with the joins along which most of them were fromulated. It may be noted that many compositions in close proximity to each other were formulated on different joins, thus contributing statistically to the over-all accuracy of the method.

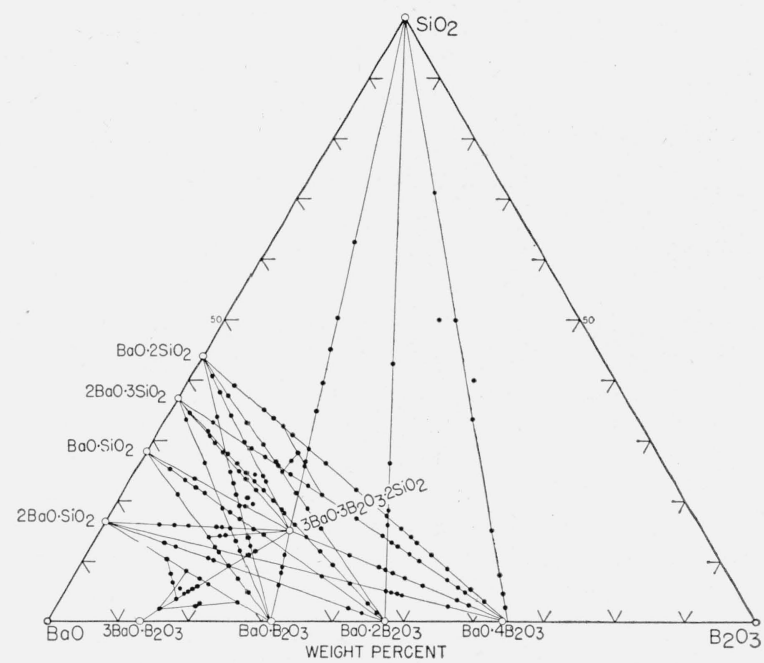

Figure 1. Compositions studied in the system $\mathrm{BaO}-\mathrm{B}_{2} \mathrm{O}_{3}-$ $\mathrm{SiO}_{2}$, together with the joins along which most of them were formulated.

It might also be emphasized that the prepared compositions always consisted of crystalline or mixtures of crystalline and glassy phases, as equilibrium conditions could be approached more rapidly by melting than by crystallization from above the liquidus.

\subsection{Apparatus and Method}

Phase equilibria relationships in the system were obtained by the well established quenching technique $[5,6]$. Charges of about $20 \mathrm{mg}$ were heated in the relatively constant temperature zone (hottest region) of a vertical tube, platinum 80 percent-rhodium 20 percent wound, resistance furnace [7]. Constanttemperature control in this zone to within $\pm 0.3 \mathrm{deg}$. $\mathrm{C}$ was achieved with the use of a newly designed, self-adjusting, a-c bridge-type controller, described later.

Temperatures were measured with platinum versus platinum-rhodium $(10 \%)$ thermocouples, which were originally calibrated against a standard thermocouple by the Pyrometry Laboratory of the National Bureau of Standards. All temperature values are given on the International Temperature Scale of 1948, based on absolute millivolts [8]. Be- 
tween $630.5^{\circ}$ and $1,063.0^{\circ} \mathrm{C}$ (the gold point) the differences between the International Temperature Scales of 1948 and 1927 are less than 0.5 deg. C. At $1,500^{\circ} \mathrm{C}$, however, the International Scale of 1948 is 2.3 deg. Cless than the scale of 1927 ; and at $2,000^{\circ} \mathrm{C}$, the difference is $6.4 \mathrm{deg}$. $\mathrm{C}$ [9]. In reviewing the literature of high-temperature phase studies, it is found that many investigators go into great detail concerning the accuracy of thermocouple calibrations, yet never specify the temperature scale used. It cannot be safely assumed that all temperature values after January 1, 1948, are reported on the International Temperature Scale of 1948, even though on that day, by international agreement, the change from international to absolute units became effective. The changeover at the National Bureau of Standards, for example, did not become effective until January 1, 1949. Since 1914 the Geophysical Laboratory of the Carnegie Institution of Washington has used a temperature scale based on the work of Day and Sosman. The scale is in good agreement with the International Temperature Scale of 1948 , up to about $1,550^{\circ} \mathrm{C}$.

The thermocouple calibrations were checked periodically against the melting points of barium tetraborate $\left(889^{\circ} \mathrm{C}\right) \quad[4]$, the ternary compound, $3 \mathrm{BaO} \cdot 3 \mathrm{~B}_{2} \mathrm{O}_{3} \cdot 2 \mathrm{SiO}_{2}\left(1,009^{\circ} \mathrm{C}\right)$, gold $\left(1,063^{\circ} \mathrm{C}\right)$, barium borate $\left(1,105^{\circ} \mathrm{C}\right)[4]$ and barium disilicate $\left(1,416^{\circ}\right.$ C). ${ }^{2}$

Quenched samples were crushed and examined with the polarizing microscope, using the standard immersion media methods. Refractive indices measured by this method are accurate to within \pm 0.003 for $n_{D}$.

X-ray diffraction powder patterns (with copper K alpha radiation) were made for a number of compounds and mixtures. A commercial type Geiger counter X-ray spectrometer equipped with a synchronous motor-drivan scanning unit and attached to an electronic high-speed recorder was used for this purpose.

\subsection{Adjusting, Bridge-type Controller}

A self-adjusting, bridge-type controller suitable for precise temperature control of a quenching furnace was designed and assembled from commercially available components by F. A. Mauer of the National Bureau of Standards. Like the electricfurnace thermostat developed by $\mathrm{H}$. S. Roberts at the Geophysical Laboratory in 1925 [7], the controller operates on the change of resistance that accompanies a change in temperature of the furnace winding. The contacting galvanometer has been replaced by a servo amplifier (A, fig. 2), which provides power to operate a reversible, two-phase motor, M. As the amplifier operates on a 60-cycle a-c signal, the power for the furnace is adjusted by means of a variable autotransformer. This transformer is positioned by the reversible motor and provides the power required to maintain the furnace winding at the temperature prescribed by the resistance setting. The temperature is maintained constant, of course,

\footnotetext{
${ }^{2}$ In accordance with the International Temperature Scale of 1948.
}

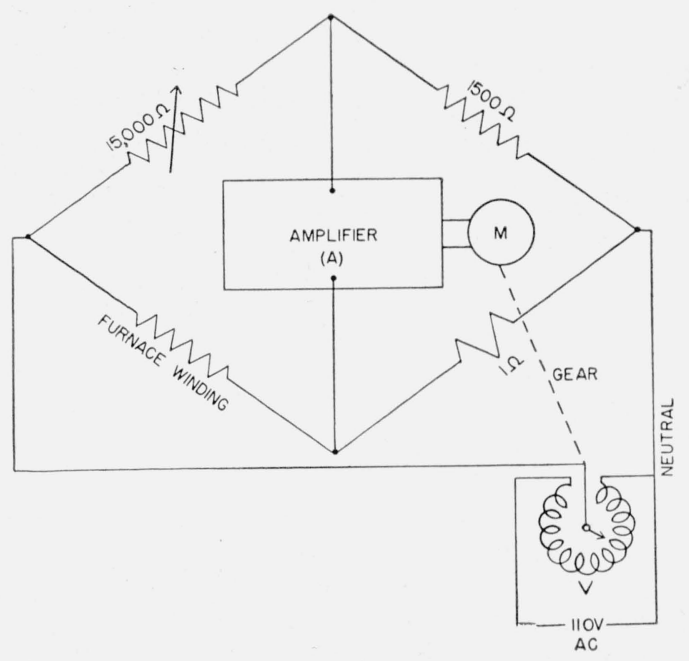

Figure 2. Schematic diagram of self-adjusting, bridge-type controller for high-temperature furnace.

A, Servo amplifier; M, reversible balancing motor, $27 \mathrm{rpm}$; V, variable autotransformer.

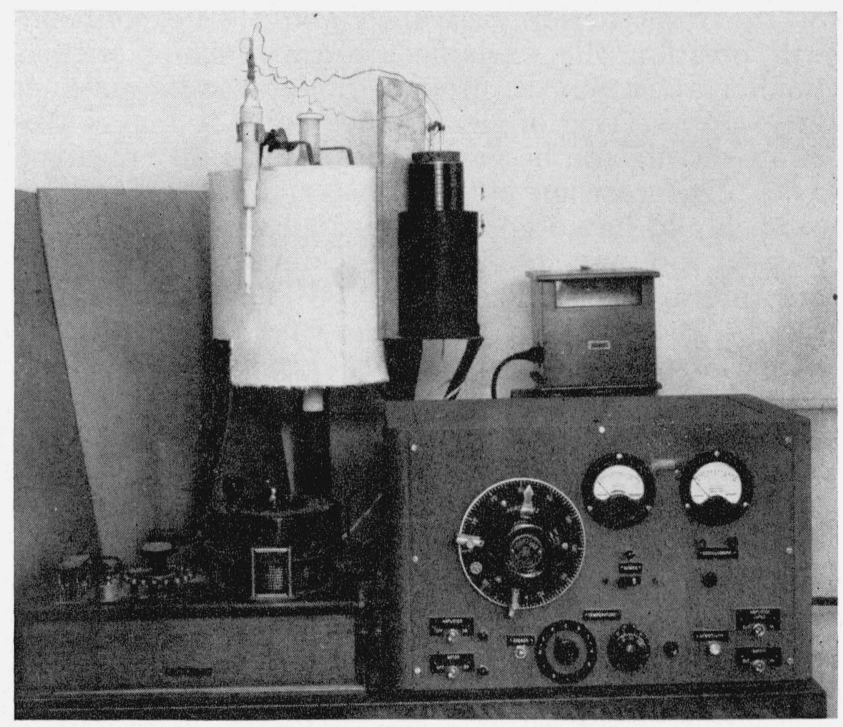

FIGURE 3 . Temperature controller together with the quenching furnace and the temperature measuring equipment.

only if volatilization of the winding is negligible.

Figure 2 shows a schematic diagram of the controller, and figure 3 shows the controller, together with the quenching furnace and the temperature measuring equipment.

The controller offers a combination of features that have not been available in any one instrument. These principal advantages are as follows:

1. Control of temperature to within $\pm 0.3 \mathrm{deg}$. C.

2. Elimination of process lags by use of the furnace winding as the sensing element.

3. Use of alternating current to heat the furnace. This feature is important because a-c power is readily available, permits the use of a variable autotransformer to adjust the voltage without 
wasting power, and also permits amplification of the signal from the bridge.

4. Incorporation of proportional speed floating control with automatic reset. When the temperature departs only slightly from the control point, the autotransformer moves at a rate proportional to the departure. In the case of a large unbalance, the motor turns at an essentially constant speed until balance is reached. Because the motor rotates as long as the temperature remains different from the required temperature, the variac automatically assumes a new position for each temperature setting and even compensates for change in the line voltage of up to 20 percent. As long as power requirements and line voltage remain constant, oscillations of the balancing motor are barely perceptible. Consequently, even if the controller is switched off, the temperature will remain within a few degrees of the control point. This characteristic simplifies the problem of controlling the furnace in a desired temperature range so that it can be ready for use on the following day.

5. Simplification of operating procedure. A single knob is used for resetting the control point. Because of the reset action described above, the controller will position the variable autotransformer without further attention, to provide the power requirement for a new setting of the balance point. The control point setting can be varied continuously by means of a 10-turn precision potentiometer.

It should be noted that since an a-c bridge circuit is used, an impedance balance must be effected if maximum precision is desired. The bridge output can be recorded on an oscilloscope and capacitors added to the high-impedance arms of the bridge until a true null can be obtained [10].

The principal maintenance problem is presented by the variable autotransformer brushes. During operation, the "hunting" of the controller keeps the brushes moving back and forth between adjacent windings. Experience indicates that after about 6 months of operation, arcing may occur between the brushes and the slightly roughened windings. In this case, the brushes are cleaned or replaced, and the windings are cleaned according to the manufacturer's instructions.

\section{Limiting Binary Systems}

\subsection{System $\mathrm{BaO}-\mathrm{B}_{2} \mathrm{O}_{3}$}

The binary system $\mathrm{BaO}-\mathrm{B}_{2} \mathrm{O}_{3}$ was reported by Levin and McCurdie [4]. It is distinguished by a region of two-liquid immiscibility extending from about 70 percent of $\mathrm{B}_{2} \mathrm{O}_{3}(30$ percent of $\mathrm{BaO})$ to almost pure $\mathrm{B}_{2} \mathrm{O}_{3}$. Extrapolation from ternary data to the binary indicated that the reported binary liquidus temperatures were uniformly low by about $10^{\circ} \mathrm{C}$. New determinations on the binary compounds confirmed this conclusion. A corrected diagram is reproduced as figure 4.

\subsection{System $\mathrm{B}_{2} \mathrm{O}_{3}-\mathrm{SiO}_{2}$}

Only limited information is available about the system $\mathrm{B}_{2} \mathrm{O}_{3}-\mathrm{SiO}_{2}$, and no liquidus values are known,

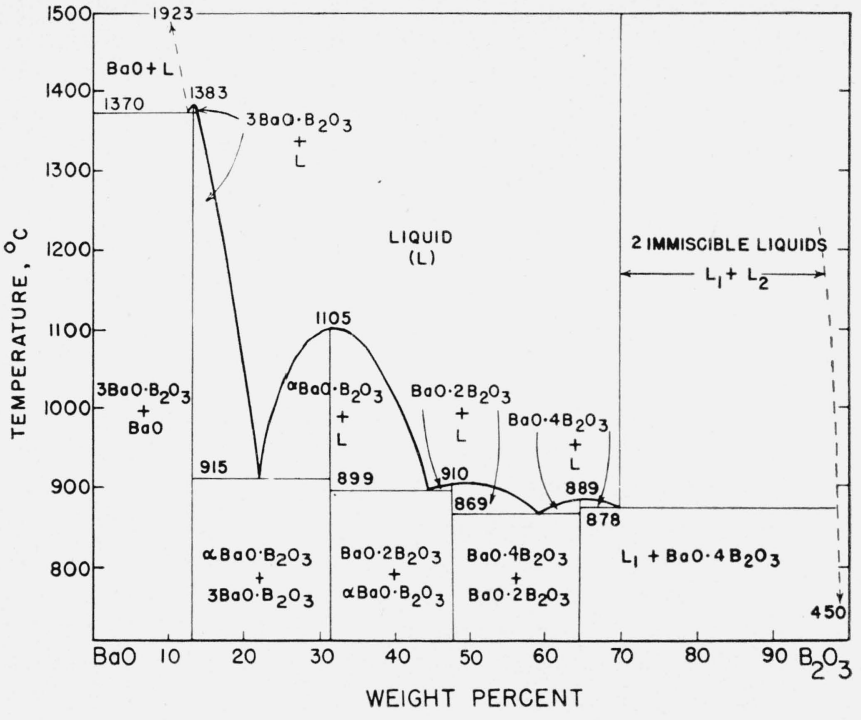

Figure 4. Phase equilibrium diagram for the system $\mathrm{BaO}-\mathrm{B}_{2} \mathrm{O}_{3}[4]$.

Liquidus values have been raised by a uniform amount of $10^{\circ} \mathrm{C}$.

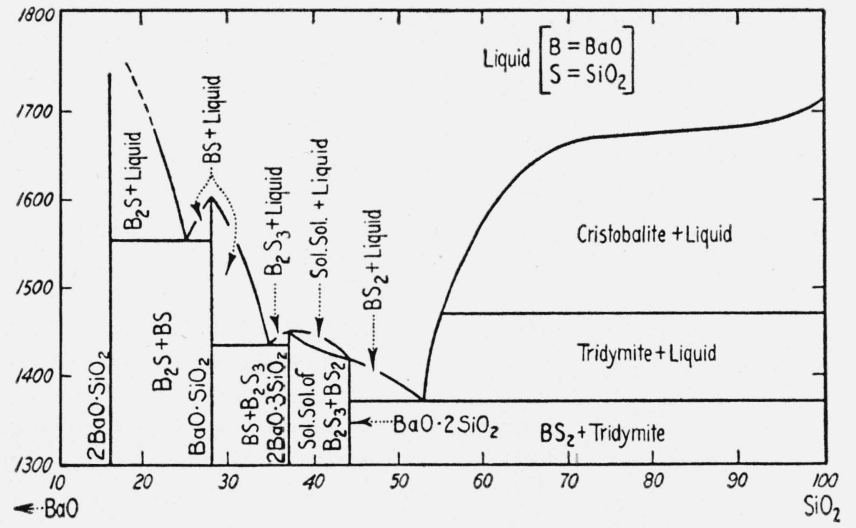

Figure 5. The Binary system $\mathrm{BaO}-\mathrm{SiO}_{2},[12,15]$. Liquidus values not changed to International temperature scale of 1948 .

as no mixture in the system has been crystallized. Morey [11] was unable to crystallize any mixture in the system, either from a dry mix or by hydrothermal treatment. Greig [12], and Cousin and Turner [13] believe that the oxides as liquids are completely miscible in all proportions. The latter authors, on the basis of thermal expansion and density-composition curves, could find no indication of compound formation. N. K. Dertev and T. H. Khudyakova [14], however, believe that several compounds (formulas unspecified) are possible.

\subsection{System $\mathrm{BaO}-\mathrm{SiO}_{2}$}

The binary system $\mathrm{BaO}-\mathrm{SiO}_{2}$, as shown in figure 5, was investigated originally by Eskola [15] and modified later by Greig [12]. It shows two regions of particular interest. In the first, dibarium trisilicate $\left(2 \mathrm{BaO} \cdot 3 \mathrm{SiO}_{2}\right)$ and barium disilicate $\left(\mathrm{BaO} \cdot 2 \mathrm{SiO}_{2}\right)$ form a complete solid solution series. The liquidus 
TABle 2. Comparison of X-ray diffraction data for compounds in the system $\mathrm{BaO}-\mathrm{SiO}_{2}$

\begin{tabular}{|c|c|c|c|c|c|c|c|c|c|c|c|c|c|c|c|}
\hline \multicolumn{4}{|c|}{$2 \mathrm{BaO} \cdot \mathrm{SiO}_{2}$} & \multicolumn{4}{|c|}{$\mathrm{BaO} \cdot \mathrm{SiO}_{2}$} & \multicolumn{4}{|c|}{$2 \mathrm{BaO} \cdot 3 \mathrm{SiO}_{2}$} & \multicolumn{4}{|c|}{$\mathrm{BaO} \cdot 2 \mathrm{SiO}_{2}$} \\
\hline \multicolumn{2}{|c|}{ (a) } & \multicolumn{2}{|c|}{ (b) } & \multicolumn{2}{|c|}{ (a) } & \multicolumn{2}{|c|}{ (b) } & \multicolumn{2}{|c|}{ (a) } & \multicolumn{2}{|c|}{ (b) } & \multicolumn{2}{|c|}{ (a) } & \multicolumn{2}{|c|}{ (b) } \\
\hline$d \mathrm{e}$ & $I^{\mathrm{d}}$ & $d$ & $I$ & $d$ & $I$ & $d$ & $I$ & $d$ & $I$ & $d$ & $I$ & $d$ & $I$ & $d$ & $I$ \\
\hline \begin{tabular}{c}
$A$ \\
\hdashline-23 \\
4.23 \\
$\cdots \cdots$
\end{tabular} & \begin{tabular}{l}
$\%$ \\
20 \\
\hdashline \\
\end{tabular} & $\begin{array}{l}\text { A } \\
5.1 \\
4.4 \\
4.23 \\
3.78 \\
3.53\end{array}$ & $\begin{array}{c}\% \\
10 \\
10 \\
19 \\
7 \\
12\end{array}$ & $\begin{array}{c}A \\
5.19 \\
4.20 \\
3.73 \\
\text { 3. } 58 \\
3.43\end{array}$ & $\begin{array}{c}\% \\
19 \\
10 \\
55 \\
17 \\
100 \\
(1)\end{array}$ & $\begin{array}{c}A \\
4.22 \\
3.70 \\
3.41\end{array}$ & $\begin{array}{c}\% \\
13 \\
40(2) \\
100(1)\end{array}$ & $\begin{array}{c}A \\
7.11 \\
6.06 \\
3.93 \\
3.79\end{array}$ & $\begin{array}{c}\% \\
15 \\
11 \\
13 \\
100(1)\end{array}$ & $\begin{array}{l}\text { A } \\
7.0 \\
6.05 \\
5.00 \\
4.33 \\
\text { 3. } 75\end{array}$ & $\begin{array}{c}\% \\
12 \\
6 \\
6 \\
6 \\
100 \\
(1)\end{array}$ & $\begin{array}{c}A \\
7.00 \\
6.87 \\
5.63 \\
4.75 \\
4.15\end{array}$ & $\begin{array}{l}\% \\
14 \\
13 \\
17 \\
12 \\
8\end{array}$ & $\begin{array}{c}A \\
6.7 \\
\cdots \\
\cdots\end{array}$ & $\begin{array}{c}\% \\
9 \\
9 \\
\end{array}$ \\
\hline $\begin{array}{l}\text { 3. } 43 \\
\text { 3. } 17 \\
\text { 3. } 11 \\
\text { 3. } 03 \\
2.95\end{array}$ & $\begin{array}{r}63 \\
16 \\
14 \\
100(1) \text { e } \\
95(2)\end{array}$ & $\begin{array}{l}3.44 \\
3.18 \\
3.12 \\
3.04 \\
2.94\end{array}$ & $\begin{array}{r}64(3) \\
29 \\
28 \\
100(1) \\
100(2)\end{array}$ & $\begin{array}{l}3.36 \\
3.13 \\
\cdots \\
2.84\end{array}$ & $\begin{array}{l}57(2) \\
50 \\
32\end{array}$ & $\begin{array}{l}3.34 \\
3.13 \\
3.02 \\
2.94 \\
2.81\end{array}$ & $\begin{array}{r}22 \\
34 \\
10 \\
8 \\
28\end{array}$ & $\begin{array}{l}3.71 \\
3.51 \\
3.44 \\
3.33 \\
3.28\end{array}$ & $\begin{array}{l}75 \\
10 \\
10 \\
87 \\
76(2)\end{array}$ & $\begin{array}{l}\text { 3. } 68 \\
\text { 3. } 43 \\
\text { 3. } 30 \\
\text { 3. } 26\end{array}$ & $\begin{array}{c}80(2) \\
6 \\
70 \\
42\end{array}$ & $\begin{array}{l}\text { 4. } 02 \\
\text { 3. } 57 \\
\text { 3. } 39 \\
\text { 3. } 36 \\
\text { 3. } 27\end{array}$ & $\begin{array}{l}77(3) \\
52 \\
14 \\
23 \\
25\end{array}$ & $\begin{array}{l}\text { 4. } 00 \\
\text { 3. } 55 \\
\cdots \\
3.28\end{array}$ & $\begin{array}{c}100(1) \\
20 \\
23\end{array}$ \\
\hline $\begin{array}{l}2.91 \\
2.69 \\
2.56 \\
2.53 \\
2.44\end{array}$ & $\begin{array}{c}82(3) \\
10 \\
8 \\
14 \\
36\end{array}$ & $\begin{array}{l}2.69 \\
2.53 \\
2.44\end{array}$ & $\begin{array}{l}18 \\
20 \\
52\end{array}$ & $\begin{array}{l}2.75 \\
2.71 \\
2.59 \\
2.36\end{array}$ & $\begin{array}{r}18 \\
23 \\
9 \\
\hdashline 31\end{array}$ & $\begin{array}{l}2.77 \\
2.59 \\
2.45 \\
2.35\end{array}$ & $\begin{array}{r}24 \\
10 \\
8 \\
25\end{array}$ & $\begin{array}{l}2.78 \\
2.61 \\
\end{array}$ & $\begin{array}{r}74 \\
8\end{array}$ & $\begin{array}{l}3.13 \\
2.99 \\
2.78 \\
2.60 \\
2.55\end{array}$ & $\begin{array}{r}35 \\
6 \\
75 \\
9 \\
6 \\
6\end{array}$ & $\begin{array}{l}\text { 3. } 15 \\
\text { 3. } 11 \\
2.86 \\
2.76 \\
2.69\end{array}$ & $\begin{array}{l}100(1) \\
87(2) \\
27 \\
40 \\
7\end{array}$ & $\begin{array}{l}3.14 \\
\cdots 2.77 \\
\cdots\end{array}$ & \begin{tabular}{c}
$60(2)$ \\
\hdashline 36 \\
\hdashline$\ldots$
\end{tabular} \\
\hline $\begin{array}{l}2.40 \\
2.30 \\
2.24 \\
2.12 \\
2.10\end{array}$ & $\begin{array}{r}25 \\
6 \\
27 \\
24 \\
36\end{array}$ & $\begin{array}{l}2.4 \\
2.31 \\
\text { 2. } 255 \\
\text { 2. } 13 \\
2.11\end{array}$ & $\begin{array}{l}32 \\
10 \\
45 \\
32 \\
35\end{array}$ & $\begin{array}{l}2.30 \\
2.24 \\
2.19 \\
2.14 \\
2.09\end{array}$ & $\begin{array}{r}35 \\
31 \\
5 \\
11 \\
19\end{array}$ & $\begin{array}{l}2.14 \\
2.09\end{array}$ & $\begin{array}{l}34 \\
11 \\
40(3)\end{array}$ & $\begin{array}{l}2.41 \\
2.36 \\
2.28 \\
2.23 \\
2.21\end{array}$ & $\begin{array}{r}8 \\
35 \\
45 \\
32 \\
24\end{array}$ & $\begin{array}{l}2.40 \\
2.35 \\
2.27 \\
2.225 \\
2.205\end{array}$ & $\begin{array}{r}9 \\
14 \\
23 \\
23 \\
13\end{array}$ & $\begin{array}{l}2.61 \\
2.36 \\
2.35 \\
2.26 \\
2.23\end{array}$ & $\begin{array}{l}12 \\
16 \\
16 \\
32 \\
29\end{array}$ & $\begin{array}{l}2.60 \\
2.35 \\
2.27 \\
2.23\end{array}$ & $\begin{array}{l}10 \\
22 \\
50 \\
50\end{array}$ \\
\hline $\begin{array}{l}\text { 2. } 02 \\
1.975 \\
\text { 1. } 909 \\
1.867\end{array}$ & $\begin{array}{c}8 \\
22 \\
17 \\
22\end{array}$ & $\begin{array}{l}2.03 \\
1.98 \\
1.94 \\
1.91 \\
1.87\end{array}$ & $\begin{array}{r}16 \\
29 \\
7 \\
24 \\
28\end{array}$ & $\begin{array}{l}2.08 \\
2.04 \\
\text { 2. } 01 \\
1.977 \\
1.896\end{array}$ & $\begin{array}{r}36 \\
45 \\
7 \\
7 \\
32\end{array}$ & $\begin{array}{l}1.99 \\
1.90\end{array}$ & $\begin{array}{l}30 \\
15 \\
29\end{array}$ & $\begin{array}{l}2.14 \\
2.09 \\
2.04 \\
1.981 \\
1.918\end{array}$ & $\begin{array}{l}60 \\
22 \\
12 \\
20 \\
14\end{array}$ & $\begin{array}{l}2.14 \\
2.08 \\
2.04 \\
1.98 \\
1.91\end{array}$ & $\begin{array}{l}41 \\
18 \\
12 \\
17 \\
14\end{array}$ & $\begin{array}{l}2.21 \\
2.16 \\
2.06 \\
2.05 \\
2.00\end{array}$ & $\begin{array}{r}51 \\
25 \\
21 \\
32 \\
7\end{array}$ & $\begin{array}{l}2.17 \\
2.06 \\
\cdots\end{array}$ & \begin{tabular}{l}
50 \\
25 \\
\hdashline
\end{tabular} \\
\hline $\begin{array}{l}\text { 1. } 788 \\
\text { 1. } 762\end{array}$ & $\begin{array}{l}10 \\
33\end{array}$ & $\begin{array}{l}1.80 \\
1.77 \\
1.73\end{array}$ & $\begin{array}{l}14 \\
49 \\
10\end{array}$ & $\begin{array}{l}\text { 1. } 852 \\
\text { 1. } 795 \\
\text { 1. } 781\end{array}$ & $\begin{array}{r}16 \\
9 \\
9\end{array}$ & $\begin{array}{l}1.86 \\
1.79\end{array}$ & $\begin{array}{l}17 \\
10\end{array}$ & 1.881 & 8 & $\begin{array}{l}1.88 \\
1.86 \\
1.825\end{array}$ & $\begin{array}{r}7 \\
7 \\
16\end{array}$ & $\begin{array}{l}\text { 1. } 923 \\
\text { 1. } 877 \\
1.827\end{array}$ & $\begin{array}{r}12 \\
17 \\
8\end{array}$ & $\begin{array}{l}1.91 \\
1.87\end{array}$ & $\begin{array}{l}15 \\
20\end{array}$ \\
\hline $\begin{array}{l}\text { 1. } 710 \\
\text { 1. } 686\end{array}$ & $\begin{array}{l}47 \\
11\end{array}$ & $\begin{array}{l}\text { 1. } 715 \\
\text { 1. } 69\end{array}$ & $\begin{array}{l}10 \\
48 \\
15\end{array}$ & $\begin{array}{l}\text { 1. } 761 \\
\text { 1. } 737\end{array}$ & $\begin{array}{r}9 \\
12 \\
12\end{array}$ & $\begin{array}{l}1.76 \\
1.72\end{array}$ & $\begin{array}{l}13 \\
14\end{array}$ & $\begin{array}{l}\text { 1. } 811 \\
\text { 1. } 789\end{array}$ & $\begin{array}{l}17 \\
25\end{array}$ & 1. 79 & 20 & $\begin{array}{l}\text { 1. } 783 \\
\text { 1. } 732\end{array}$ & $\begin{array}{r}16 \\
9\end{array}$ & 1.78 & 12 \\
\hline $\begin{array}{l}\text { 1. } 659 \\
\text { 1. } 645 \\
\text { 1. } 598 \\
\text { 1. } 578 \\
\text { 1. } 554\end{array}$ & $\begin{array}{l}7 \\
8 \\
5 \\
3 \\
3 \\
3\end{array}$ & $\begin{array}{l}\text { 1. } 66 \\
\text { 1. } 64 \\
\text { 1. } 605 \\
\text { 1. } 58 \\
\text {-..- }\end{array}$ & $\begin{array}{r}9 \\
25 \\
8 \\
7\end{array}$ & $\begin{array}{l}1.698 \\
1.635 \\
1.606 \\
1.570 \\
1.545\end{array}$ & $\begin{array}{r}33 \\
4 \\
7 \\
10 \\
7\end{array}$ & $\begin{array}{l}\ldots . . \\
1.61 \\
1.56 \\
\ldots . .\end{array}$ & $\begin{array}{l}7 \\
9\end{array}$ & $\begin{array}{l}\text { 1. } 760 \\
\text { 1. } 745 \\
\text { 1. } 654 \\
\text { 1. } 636 \\
\text { 1. } 617\end{array}$ & $\begin{array}{r}11 \\
11 \\
22 \\
10 \\
7\end{array}$ & $\begin{array}{l}\text { 1. } 76 \\
\text { 1. } 71 \\
1.65 \\
1.63 \\
1.615\end{array}$ & $\begin{array}{r}16 \\
8 \\
12 \\
6 \\
6\end{array}$ & $\begin{array}{l}\text { 1. } 706 \\
1.601 \\
1.584 \\
1.571 \\
1.481\end{array}$ & $\begin{array}{r}31 \\
13 \\
9 \\
15 \\
8\end{array}$ & $\begin{array}{l}\ldots \\
1.59 \\
1.48\end{array}$ & 10 \\
\hline $\begin{array}{l}\text { 1. } 511 \\
\text { 1. } 470 \\
\text { 1. } 455 \\
\text { 1. } 442\end{array}$ & $\begin{array}{r}6 \\
4 \\
5 \\
5 \\
12\end{array}$ & $\begin{array}{l}\text { 1. } 52 \\
\text { 1. } 51 \\
\text { 1. } 475 \\
1.46 \\
1.445\end{array}$ & $\begin{array}{r}12 \\
13 \\
8 \\
12 \\
12\end{array}$ & $\begin{array}{l}\text { 1. } 517 \\
\text { 1. } 486 \\
\text { 1. } 477 \\
\text { 1. } 468 \\
1.448\end{array}$ & $\begin{array}{r}13 \\
8 \\
8 \\
12 \\
10\end{array}$ & \begin{tabular}{l}
1.525 \\
\hdashline$\cdots$ \\
\hdashline$\cdots$ \\
$\cdots$
\end{tabular} & $\begin{array}{c}11 \\
\\
\end{array}$ & $\begin{array}{l}1.557 \\
1.518 \\
1.513\end{array}$ & $\begin{array}{r}11 \\
7 \\
8\end{array}$ & $\begin{array}{l}\text { 1. } 60 \\
\text { 1. } 58 \\
1.555 \\
\text { 1. } 52\end{array}$ & $\begin{array}{l}6 \\
6 \\
8 \\
8\end{array}$ & $\begin{array}{l}1.458 \\
1.408 \\
1.392 \\
1.378 \\
1.292\end{array}$ & $\begin{array}{r}11 \\
6 \\
6 \\
9 \\
16\end{array}$ & \begin{tabular}{l}
1.46 \\
\hdashline$\cdots$ \\
1.29
\end{tabular} & $\begin{array}{c}10 \\
10\end{array}$ \\
\hline $\begin{array}{l}\text { 1. } 432 \\
\text { 1. } 416 \\
\text { 1. } 370 \\
\text { 1. } 338\end{array}$ & $\begin{array}{c}10 \\
7 \\
10 \\
5\end{array}$ & $\begin{array}{l}1.435 \\
1.42 \\
1.375 \\
1.345 \\
\ldots . .\end{array}$ & $\begin{array}{r}16 \\
9 \\
20 \\
12 \\
\end{array}$ & $\begin{array}{l}\text { 1. } 430 \\
\text { 1. } 415 \\
\text { 1. } 392 \\
\text { 1. } 366 \\
\text { 1. } 333\end{array}$ & $\begin{array}{r}11 \\
8 \\
10 \\
10 \\
9\end{array}$ & $\begin{array}{l}\ldots . \\
1.40 \\
1.375 \\
\ldots . .\end{array}$ & $\begin{array}{c}10 \\
9\end{array}$ & $\begin{array}{l}1.505 \\
1.481 \\
1.474 \\
1.450 \\
1.422\end{array}$ & $\begin{array}{r}6 \\
6 \\
10 \\
6 \\
11\end{array}$ & $\begin{array}{l}\text { 1. } 505 \\
1.48 \\
\cdots 1.4 \\
1.42\end{array}$ & $\begin{array}{r}6 \\
6 \\
\\
8\end{array}$ & $\begin{array}{l}\text { 1. } 288 \\
\text { 1. } 285 \\
\text { 1. } 281 \\
\cdots\end{array}$ & $\begin{array}{l}16 \\
10 \\
10 \\
\end{array}$ & $\begin{array}{l}\cdots \\
\cdots \cdots \\
\cdots \cdots \\
\cdots \\
\cdots\end{array}$ & w... \\
\hline $\begin{array}{l}\text { 1. } 315 \\
\text { 1. } 292 \\
\text { 1. } 263 \\
\text { 1. } 248 \\
-.-\end{array}$ & $\begin{array}{r}10 \\
5 \\
10 \\
10 \\
\end{array}$ & $\begin{array}{l}1.32 \\
1.28 \\
1.265 \\
1.25 \\
1.24\end{array}$ & $\begin{array}{l}25 \\
16 \\
23 \\
15 \\
11\end{array}$ & $\begin{array}{l}\text { 1. } 315 \\
\text { 1. } 306 \\
\text { 1. } 291 \\
\text { 1. } 279 \\
\text { 1. } 270\end{array}$ & $\begin{array}{r}10 \\
7 \\
7 \\
12 \\
8\end{array}$ & $\begin{array}{l}1.315 \\
1.28 \\
1.28\end{array}$ & \begin{tabular}{c}
8 \\
\hdashline 5
\end{tabular} & $\begin{array}{l}\text { 1. } 406 \\
\text { 1. } 394 \\
\text { 1. } 350 \\
\text { 1. } 342 \\
\text { 1. } 322\end{array}$ & $\begin{array}{r}4 \\
31 \\
8 \\
6 \\
13\end{array}$ & $\begin{array}{l}1.395 \\
1.35 \\
1.33 \\
1.32\end{array}$ & $\begin{array}{r}14 \\
6 \\
8 \\
6\end{array}$ & $\begin{array}{l}\ldots . . \\
\cdots \cdots \\
\cdots \cdots \\
\cdots \\
\cdots . .\end{array}$ & $\begin{array}{l} \\
\ldots\end{array}$ & $\begin{array}{l}\cdots \\
\cdots \\
\cdots \\
\cdots \\
\cdots\end{array}$ & $\begin{array}{l}\mathrm{n} \\
\cdots \\
\cdots\end{array}$ \\
\hline \begin{tabular}{l}
$\cdots . .$. \\
$\cdots \cdots$. \\
\hdashline$\cdots .$. \\
$\cdots \cdots$
\end{tabular} & $\begin{array}{l}\ldots \ldots \\
\cdots \cdots \\
\cdots \cdots \\
\cdots \cdots\end{array}$ & $\begin{array}{l}1.215 \\
1.19 \\
1.17 \\
1.155 \\
1.14\end{array}$ & $\begin{array}{r}8 \\
21 \\
11 \\
25 \\
7\end{array}$ & $\begin{array}{l}\ldots . . \\
\cdots \cdots \\
\cdots \cdots \\
\cdots\end{array}$ & 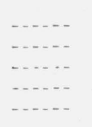 & $\begin{array}{l}1.24 \\
1.15 \\
\cdots \\
\cdots\end{array}$ & 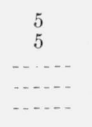 & $\begin{array}{l}\text { 1. } 299 \\
\text { 1. } 249 \\
\text { 1. } 241 \\
\text { 1. } 225 \\
\text { 1. } 200\end{array}$ & $\begin{array}{r}10 \\
5 \\
7 \\
5 \\
7\end{array}$ & $\begin{array}{l}1.30 \\
1.25 \\
1.24 \\
1.22 \\
1.20\end{array}$ & $\begin{array}{r}15 \\
9 \\
6 \\
6 \\
8\end{array}$ & $\begin{array}{l}\ldots . . \\
\cdots \cdot . \\
\cdots . . \\
\cdots . .\end{array}$ & 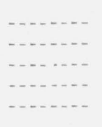 & $\begin{array}{l}\cdots \cdot . \\
\cdots \cdots \\
\cdots \cdots \\
\cdots\end{array}$ & $\begin{array}{l}\cdots \cdots \\
\cdots \cdots \\
\cdots \cdots \\
\cdots \cdots\end{array}$ \\
\hline …. & ........ & $\cdots$ & ........ & $\ldots$ & (....... & 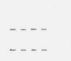 & ....... & .... & ....... & $\begin{array}{l}1.17 \\
1.135\end{array}$ & $\begin{array}{l}6 \\
9\end{array}$ & $\ldots$ & (n...... & $\cdots$ & -..... \\
\hline
\end{tabular}

a Data from present investigation. b Data by A. E. Austin [20]. - $d=$ interplanar spacing.

curve shows no maximum or minimum.

d $I=$ relative intensity.

- $(1),(2),(3)=$ three strongest peaks in order of intensity. 16] concluded from his investigation of the system $\mathrm{BaO}-\mathrm{Al}_{2} \mathrm{O}_{3}-\mathrm{SiO}_{2}$ that dibarium trisilicate is not a compound at all but is merely that particular composition at or near the maximum in a solid solution series.

Another point of interest is the shape of the liquidus curve in the cristobalite primary phase area [12]. Indicative of an approach to two-liquid immiscibility, the curve is almost horizontal for a change in composition of nearly 30 percent. Ol'shanskiû[17] investi- gated the system $\mathrm{BaO}-\mathrm{SiO}_{2}$ above the liquidus, between the temperatures of $1,800^{\circ}$ and $2,570^{\circ} \mathrm{C}$, but could find no trace of immiscible liquids. However, the S-shaped form of the portion of the liquids curve under discussion is typical of unmixing, or of a miscibility gap, occurring completely below the liquidus [18].

Before investigating the interior of the ternary system, it was considered desirable from the standpoints of experience and of possible additional information that might be gained, to check several of the 
TABLE 3. Data on liquidus determinations in the system $\mathrm{BaO}-\mathrm{B}_{2} \mathrm{O}_{3}-\mathrm{SiO}_{2}$

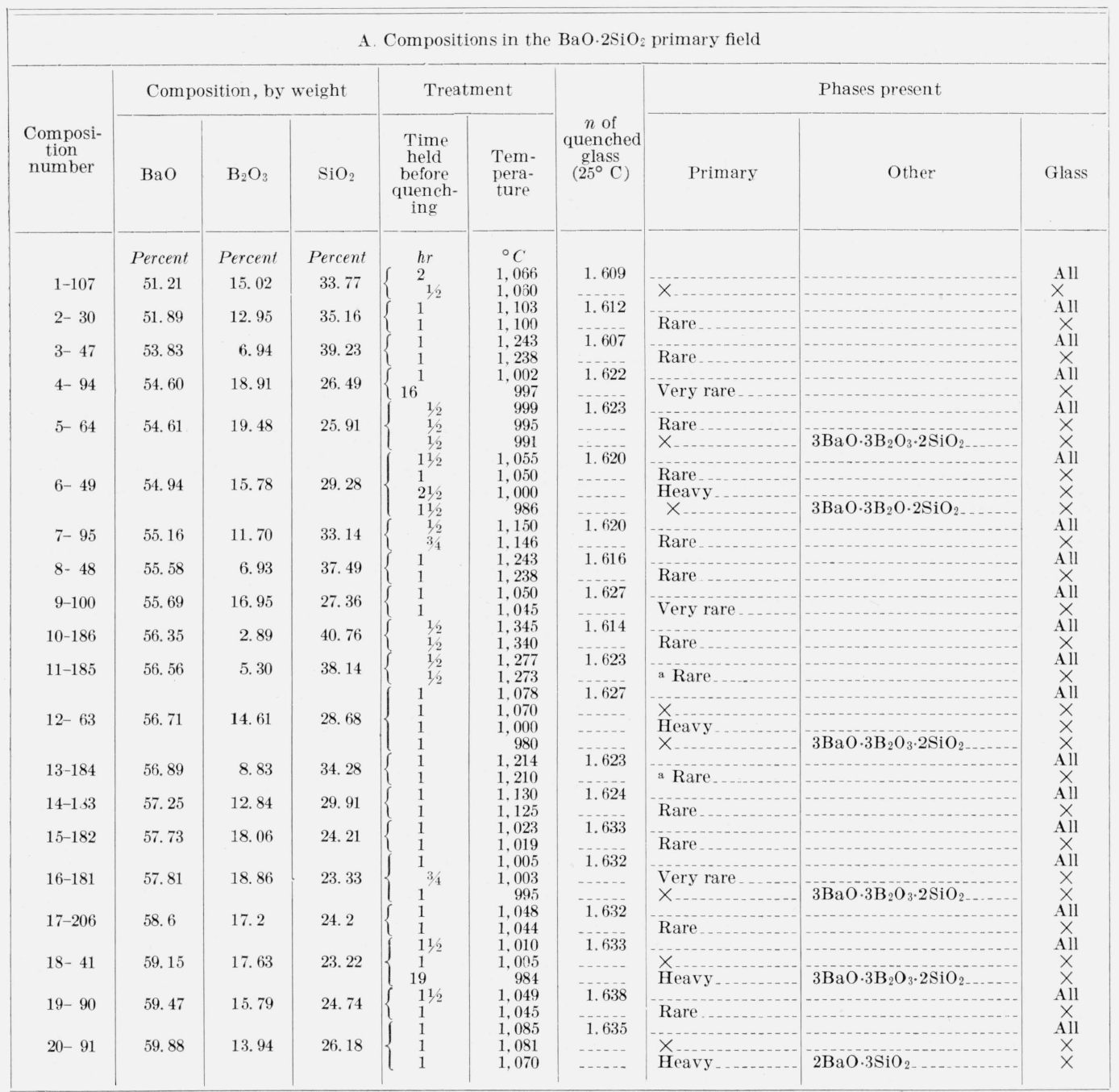

B. Compositions in the $1 \mathrm{BaO} \cdot 3 \mathrm{SiO}_{2}$ primary field and solid-solution area

\begin{tabular}{|c|c|c|c|c|c|c|c|c|c|}
\hline $21-51$ & 59.02 & 6.97 & 34.01 & $\begin{array}{l}1 \\
1\end{array}$ & $\begin{array}{l}\text { 1. } 238 \\
\text { 1. } 233\end{array}$ & 1. 626 & b ss & & All \\
\hline $22-62$ & 59.98 & 7.01 & 33.01 & $\begin{array}{l}1 / 2 \\
1 / 2\end{array}$ & 1,244 & 1. 630 & & & All \\
\hline $23-175$ & 60.21 & 16.16 & 23.63 & $1_{1,6}$ & $\begin{array}{l}1,042 \\
1,037\end{array}$ & 1. 638 & Rare & & All \\
\hline $24-26$ & 60.41 & 11.83 & 27.76 & $1_{34}$ & $\begin{array}{l}1,125 \\
1,120\end{array}$ & 1. 638 & Bare & & $\underset{x}{\text { All }}$ \\
\hline $25-176$ & 60.49 & 14. 53 & 24.98 & $\begin{array}{l}3 / 4 \\
3 / 4\end{array}$ & $\begin{array}{l}\text { 1., } 073 \\
1,068\end{array}$ & 1. 633 & "Rare & & All \\
\hline $26-177$ & 61.13 & 10.81 & 28.06 & $\begin{array}{l}1 \\
1 \\
1 / 2\end{array}$ & $\begin{array}{l}1,152 \\
1,147 \\
1,000\end{array}$ & 1. 638 & $\cdot x$ & & $\begin{array}{l}\text { All } \\
\times \\
\text { All }\end{array}$ \\
\hline $27-202$ & 61.42 & 18.13 & 20.45 & $\begin{array}{l}1 \\
1 \\
1\end{array}$ & $\begin{array}{l}999 \\
991 \\
981\end{array}$ & - & $\begin{array}{l}\text { Very rare } \\
\text { Moderate } \\
\text { Heavy }\end{array}$ & $3 \mathrm{~B} 3 \mathrm{O} \cdot 3 \mathrm{~B}_{2} \mathrm{O}_{3} \cdot 2 \mathrm{SiO}_{2}$ & $\begin{array}{l}x \\
x \\
x\end{array}$ \\
\hline $28-178$ & 61.46 & 8.90 & 29.64 & $\begin{array}{l}1 / 4 \\
1 / 4\end{array}$ & $\begin{array}{l}1,201 \\
1,196\end{array}$ & 1. 638 & & & $\stackrel{\text { All }}{\times}$ \\
\hline $29-42$ & 61.47 & 6. 96 & 31.57 & $\begin{array}{l}1 \\
1\end{array}$ & $\begin{array}{l}1,245 \\
1,240\end{array}$ & 1. 638 & b ss & & All \\
\hline 30-179 & 61.68 & 7. 63 & 30.69 & $\begin{array}{l}1 \\
1\end{array}$ & $\begin{array}{l}1,226 \\
1,223\end{array}$ & 1. 638 & & & $\underset{x}{\text { All }}$ \\
\hline $31-54$ & 61.74 & 13.40 & 24.86 & $1^{1 / 2}$ & $\begin{array}{l}1,089 \\
1,085\end{array}$ & 1. 640 & Rare & & $\stackrel{\text { All }}{x}$ \\
\hline 32- 79 & 62.29 & 14. 71 & 23.00 & $1^{3 / 4}$ & $\begin{array}{l}1,064 \\
1,061\end{array}$ & 1. 642 & Rare & & $\stackrel{\text { All }}{x}$ \\
\hline $33-180$ & 62.49 & 2. 90 & 34.61 & ]$_{1 / 2}^{1 / 2}$ & $\begin{array}{l}1,365 \\
1,360\end{array}$ & 1. 637 & & & All \\
\hline $34-172$ & 62.53 & 18.30 & 19.17 & 1 & $\begin{array}{l}990 \\
980\end{array}$ & $1.0+1$ & $\underset{\times}{\text { Rare }}$ & $3 \mathrm{BaO} 3 \mathrm{~B}_{2} \mathrm{O} 3 \cdot 2 \mathrm{SiO}_{2}$ & $x$ \\
\hline $35-52$ & 62.87 & 16.08 & 21.05 & $\begin{array}{l}11 / 2 \\
11 / 4\end{array}$ & $\begin{array}{l}1,040 \\
1,035\end{array}$ & 1.642 & Ware & ... & $\stackrel{A 11}{x}$ \\
\hline
\end{tabular}

See footnotes at end of table. 
TABLE 3. Data on liquidus determinations in the system $\mathrm{BaO}-\mathrm{B}_{2} \mathrm{O}_{3}-\mathrm{SiO}_{2}$-Continued

\begin{tabular}{|c|c|c|c|c|c|c|c|c|c|}
\hline \multirow[b]{2}{*}{$\begin{array}{c}\text { Composi- } \\
\text { tion } \\
\text { number }\end{array}$} & \multicolumn{3}{|c|}{ Composition, by weight } & \multicolumn{2}{|c|}{ Treatment } & \multirow[b]{2}{*}{$\begin{array}{c}n \text { of } \\
\text { quenched } \\
\text { glass } \\
\left(25^{\circ} \mathrm{C}\right)\end{array}$} & \multicolumn{3}{|c|}{ Phases present } \\
\hline & $\mathrm{BaO}$ & $\mathrm{B}_{2} \mathrm{O}_{3}$ & $\mathrm{SiO}_{2}$ & $\begin{array}{l}\text { Time } \\
\text { held } \\
\text { before } \\
\text { quench- } \\
\text { ing }\end{array}$ & $\begin{array}{l}\text { Tem- } \\
\text { pera- } \\
\text { ture }\end{array}$ & & Primary & Other & Glass \\
\hline & Percent & Prrcent & Percent & $\begin{array}{c}h r \\
2\end{array}$ & $\begin{array}{l}{ }^{\circ} \mathrm{C} \\
1,001\end{array}$ & 1.645 & & & All \\
\hline $36-78$ & 63.42 & 17.38 & 19. 20 & 1 & $\begin{array}{r}998 \\
.350\end{array}$ & 1. 640 & Ware & & $\stackrel{x}{x}$ \\
\hline $37-58$ & 63.59 & 3.05 & 33.36 & 1 & $\begin{array}{l}1,500 \\
1,345\end{array}$ & 1.040 & b ss & & An \\
\hline $38-59$ & 64.80 & 9.13 & 26.07 & $\begin{array}{l}1 \\
1 \\
11\end{array}$ & $\begin{array}{l}1,182 \\
1,177 \\
1\end{array}$ & $\begin{array}{r}1.645 \\
1.648\end{array}$ & Rare & & $\begin{array}{l}\text { All } \\
\times \\
x\end{array}$ \\
\hline $39-83$ & 64.92 & 16. 95 & 18. 13 & $\begin{array}{l}2 \\
11 / 2 \\
1\end{array}$ & $\begin{array}{r}998 \\
984 \\
968\end{array}$ & 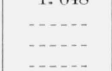 & $\begin{array}{l}\text { Rare } \\
\text { Moderate } \\
\text { Heavy }\end{array}$ & $3 \mathrm{BaO} 3 \mathrm{~B}_{2} \mathrm{O}_{3} \cdot 2 \mathrm{SiO}_{2}$ & $\begin{array}{l}\times \\
\times \\
\times\end{array}$ \\
\hline $40-60$ & 66.01 & 15. 21 & 18. 78 & $\begin{array}{l}1 \\
1\end{array}$ & $\begin{array}{l}1,036 \\
1,031\end{array}$ & 1. 649 & Rare & & $\stackrel{\text { All }}{\times}$ \\
\hline $41-76$ & 66.62 & 18.25 & 15. 13 & $1_{1}^{1 / 2}$ & $\begin{array}{l}976 \\
973\end{array}$ & 1. 654 & Rare & & $\stackrel{\text { All }}{x}$ \\
\hline $42-55$ & 67.15 & 11. 62 & 21.23 & $\begin{array}{l}1 \\
1\end{array}$ & $\begin{array}{l}1,102 \\
1,097\end{array}$ & 1. 654 & Rare & & $\stackrel{\text { Aill }}{\times}$ \\
\hline $43-173$ & 67. 22 & 9. 10 & 23.68 & $\begin{array}{l}1 / 2 \\
1 / 2\end{array}$ & $\begin{array}{l}1,164 \\
1,159\end{array}$ & 1. 654 & Very rare & & All \\
\hline $44-187$ & 67.52 & 18.13 & 14.35 & $\begin{array}{l}32 \\
3 / 4 \\
3 / 4\end{array}$ & $\begin{array}{r}103 \\
947 \\
943\end{array}$ & 1. 661 & Rare & & All \\
\hline $45-171$ & 67.65 & 16.67 & 15.68 & $\begin{array}{l}1 \\
1\end{array}$ & $\begin{array}{l}943 \\
982 \\
977\end{array}$ & 1. 668 & & & Âll \\
\hline
\end{tabular}

C. Compositions in the $\mathrm{BaO} \cdot \mathrm{SiO}_{2}$ primary field

\begin{tabular}{|c|c|c|c|c|c|c|c|c|c|}
\hline $46-84$ & 67.81 & 9.88 & 22.31 & $\begin{array}{l}1 / 2 \\
1 / 6\end{array}$ & $\begin{array}{l}1,145 \\
1,141\end{array}$ & 1.666 & Bore & & $\underset{x}{A l l}$ \\
\hline $47-127$ & 68.48 & 15.81 & 15.71 & $1^{1 / 2}$ & $\begin{array}{r}995 \\
989\end{array}$ & 1. 659 & Mare & & Âll \\
\hline $48-188$ & 68.57 & 17.19 & 14.24 & 1 & 965 & 1. 670 & $x \ldots$ & & All \\
\hline & & & & 1 & $\begin{array}{r}961 \\
1,237\end{array}$ & 1. 667 & Rare.. & & \\
\hline 49-85 & 68.61 & 7.94 & 23.45 & 1 & 1,232 & & Rare ..... & & \\
\hline $50-86$ & 69.88 & 4. 84 & 25.28 & $2^{1 / 2}$ & $\begin{array}{r}1,352 \\
964\end{array}$ & $\begin{array}{r}\sim 1.67 \\
1.664\end{array}$ & Very rare & & $\begin{array}{c}x \\
\text { All }\end{array}$ \\
\hline $51-31$ & 70.02 & 17. 68 & 12.30 & $\begin{array}{l}11 / 4 \\
11 / 2 \\
1\end{array}$ & $\begin{array}{l}960 \\
950 \\
343\end{array}$ & - & $\begin{array}{l}\text { Rare } \\
\text { Moderate } \\
\text { Moderate }\end{array}$ & $\mathrm{B}{ }_{9} \mathrm{O} \cdot \mathrm{B}_{2} \mathrm{O}_{3}$ & $\begin{array}{l}x \\
x \\
x\end{array}$ \\
\hline $52-22$ & 70.22 & 15.71 & 14.07 & $1_{1 / 2}$ & $\begin{array}{l}1,016 \\
1,013\end{array}$ & 1. 665 & Rare .. & & All \\
\hline $53-23$ & 71.04 & 7.86 & 21.10 & $\begin{array}{l}1 \\
1\end{array}$ & $\begin{array}{l}1,305 \\
1,298\end{array}$ & 1. 669 & & & Âl \\
\hline $54-168$ & 72.01 & 17. 69 & 10.30 & $\begin{array}{l}1 / 2 \\
2 / 3\end{array}$ & $\begin{array}{l}975 \\
970\end{array}$ & 1. 672 & Rare. & & $\stackrel{\text { All }}{x}$ \\
\hline $55-126$ & 72.08 & 12. 05 & 15.87 & $1^{1 / 2}$ & $\begin{array}{l}1,189 \\
1,184\end{array}$ & 1.683 & Rare ... & & All \\
\hline $56-125$ & 74. 13 & 9. 91 & 15. 96 & $\begin{array}{l}1 / 2 \\
1 / 2\end{array}$ & $\begin{array}{l}1,250 \\
1,244\end{array}$ & 1. 681 & Rare .. & & $\stackrel{\text { All }}{x}$ \\
\hline $57-70$ & 74. 93 & 18.16 & 6.91 & $1_{1 / 2}^{1}$ & $\begin{array}{l}961 \\
959\end{array}$ & - & & & $\stackrel{\text { All }}{\times}$ \\
\hline $58-169$ & 75.69 & 12.08 & 12. 23 & $\begin{array}{l}1 / 2 \\
1 / 2\end{array}$ & $\begin{array}{l}1,160 \\
1,155\end{array}$ & -...... & Very rare & & $\stackrel{\text { All }}{\times}$ \\
\hline 59-110 & 76.06 & 18. 24 & 5. 70 & $\begin{array}{l}1 \\
1\end{array}$ & $\begin{array}{l}950 \\
946\end{array}$ & 1. 684 & Rare. & & $\stackrel{\text { All }}{\times}$ \\
\hline $60-37$ & 76.11 & 15.70 & 8. 19 & $\begin{array}{l}3 / 4 \\
3 / 4\end{array}$ & $\begin{array}{l}1,038 \\
1,030\end{array}$ & 1.680 & Rare .... & & $\stackrel{\text { All }}{x}$ \\
\hline $61-158$ & 76.83 & 17.88 & 5. 29 & $\begin{array}{l}1 \\
1\end{array}$ & $\begin{array}{l}954 \\
949\end{array}$ & 1.696 & $x$ & & $\stackrel{\text { All }}{\times}$ \\
\hline $62-157$ & 77.61 & 17. 53 & 4.86 & $1^{1 / 2}$ & $\begin{array}{l}959 \\
954 \\
962\end{array}$ & $\begin{array}{r}1.695 \\
1.698\end{array}$ & Rare.... & & $\begin{array}{c}\text { All } \\
\stackrel{x}{\text { All }}\end{array}$ \\
\hline $63-200$ & 78.02 & 17. 34 & 4.64 & $\begin{array}{l}1 \\
1\end{array}$ & $\begin{array}{l}957 \\
952\end{array}$ & -..... & $\begin{array}{l}\text { Rare } \\
\text { Moderate }\end{array}$ & $3 \mathrm{BaO} \cdot \mathrm{B}_{2} \mathrm{O}_{3}$ & $\stackrel{x}{x}$ \\
\hline
\end{tabular}

D. Compositions in the $2 \mathrm{BaO} \cdot \mathrm{SiO}_{2}$ primary field

\begin{tabular}{|c|c|c|c|c|c|c|c|c|c|}
\hline $64-124$ & 76.01 & 7.95 & 16.04 & $\begin{array}{l}1 / 2 \\
1 / 2\end{array}$ & $\begin{array}{l}1,308 \\
1,302\end{array}$ & $\sim 1.69$ & Rare.. & & ${ }^{\mathrm{d}} \underset{\times}{\mathrm{All}}$ \\
\hline & & & & 1 & $\begin{array}{l}1,263 \\
1,258\end{array}$ & 1.696 & & & Âl \\
\hline $65-170$ & 76.71 & 10.54 & 12. 75 & $\begin{array}{l}1 / 2 \\
116\end{array}$ & $\begin{array}{l}1,200 \\
1,200\end{array}$ & (n..... & Moderate & & $x$ \\
\hline $66-71$ & 77.99 & 11.78 & 10.23 & $1^{1 / 2}$ & $\begin{array}{l}1,160 \\
1,224\end{array}$ & $\sim 1.694$ & Moderate & $\mathrm{BaO} \cdot \mathrm{SiO}_{2 \ldots}$ & d All \\
\hline 67-201 & 78.40 & 16.12 & $5,48,-5$ & $1^{1 / 2}$ & $\begin{array}{l}1,000 \\
1,055\end{array}$ & 1.698 & $x-$ & & All \\
\hline $01-201$ & & 10. 16 & & $\begin{array}{l}1 \\
1\end{array}$ & $\begin{array}{l}1,050 \\
1,274\end{array}$ & 1.704 & Rare _... & & uㅣ \\
\hline $68-156$ & 78.72 & 13.43 & 7.85 & 1 & 1,270 & $1.10 t$ & $\times$ & 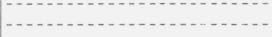 & $\times$ \\
\hline $69-155$ & 79.16 & 14.43 & 6.41 & $\begin{array}{l}2 \\
1\end{array}$ & $\begin{array}{l}1,223 \\
1,217\end{array}$ & $\begin{array}{r}1.704 \\
-\end{array}$ & Rare..... & & $\stackrel{\text { All }}{\times}$ \\
\hline $70-72$ & 79.39 & 8.84 & 11.77 & $\begin{array}{l}1 / 2 \\
1 / 2\end{array}$ & $\begin{array}{r}\text { e } 1,359 \\
1,344\end{array}$ & -...... & $\begin{array}{l}\text { Moderate } \\
\text { Heavy }\end{array}$ & & ${ }^{d} \times$ \\
\hline
\end{tabular}

See footnotes at end of table. 
TABLE 3. Data on liquidus determinations in the system $\mathrm{BaO}-\mathrm{B}_{2} \mathrm{O}_{3}-\mathrm{SiO}_{2}-\mathrm{Continued}$

\begin{tabular}{|c|c|c|c|c|c|c|c|c|c|}
\hline \multicolumn{10}{|c|}{ E. Compositions in the $3 \mathrm{BaO} \cdot \mathrm{B}_{2} \mathrm{O}_{3}$ primary field } \\
\hline \multirow[b]{2}{*}{$\begin{array}{c}\text { Composi- } \\
\text { tion } \\
\text { number }\end{array}$} & \multicolumn{3}{|c|}{ Composition, by weight } & \multicolumn{2}{|c|}{ Treatment } & \multirow[b]{2}{*}{$\begin{array}{c}n \text { of } \\
\text { quenched } \\
\text { glass } \\
\left(25^{\circ} \mathrm{C}\right)\end{array}$} & \multicolumn{3}{|c|}{ Phases present } \\
\hline & $\mathrm{BaO}$ & $\mathrm{B}_{2} \mathrm{O}_{3}$ & $\mathrm{SiO}_{2}$ & $\begin{array}{l}\text { Time } \\
\text { held } \\
\text { before } \\
\text { quench- } \\
\text { ing }\end{array}$ & $\begin{array}{l}\text { Tem- } \\
\text { pera- } \\
\text { ture }\end{array}$ & & Primary & Other & Glass \\
\hline \multirow[b]{2}{*}{$71-199$} & Percent & Percent & Percent & $h r$ & ${ }^{\circ} \mathrm{C}$ & 1698 & & & \multirow{4}{*}{$\begin{array}{l}\text { All } \\
\times \\
\times \\
\times \\
\text { All } \\
\times \\
\text { g All } \\
\text { b }\end{array}$} \\
\hline & 77.82 & 19. 76 & 2.42 & $1_{1}^{1 / 2}$ & $\begin{array}{r}906 \\
900 \\
\text { f } 875\end{array}$ & $\begin{array}{l}1.080 \\
-\cdots\end{array}$ & $\begin{array}{l}\text { Very rare } \\
\text { Moderate } \\
\text { Heavy. }\end{array}$ & $\begin{array}{l}\mathrm{Rare} \mathrm{BaO}^{\mathrm{B}_{2} \mathrm{O}_{3}} \\
\left(\mathrm{BaO} \cdot \mathrm{B}_{2} \mathrm{O}_{3}\right)\left(\mathrm{BaO} \cdot \mathrm{SiO}_{2}\right)\end{array}$ & \\
\hline $72-109$ & 80.04 & 16. 42 & 3. 54 & $1^{3 / 4}$ & 1,077 & 1.705 & & & \\
\hline $73-108$ & 83.05 & 15.05 & 1. 90 & $\begin{array}{l}11 / 2 \\
1 / 2\end{array}$ & $\begin{array}{l}1,206 \\
1,198\end{array}$ & (n) & & & \\
\hline
\end{tabular}

F. Compositions in the $\mathrm{BaO} \cdot \mathrm{B}_{2} \mathrm{O}_{3}$ primary field

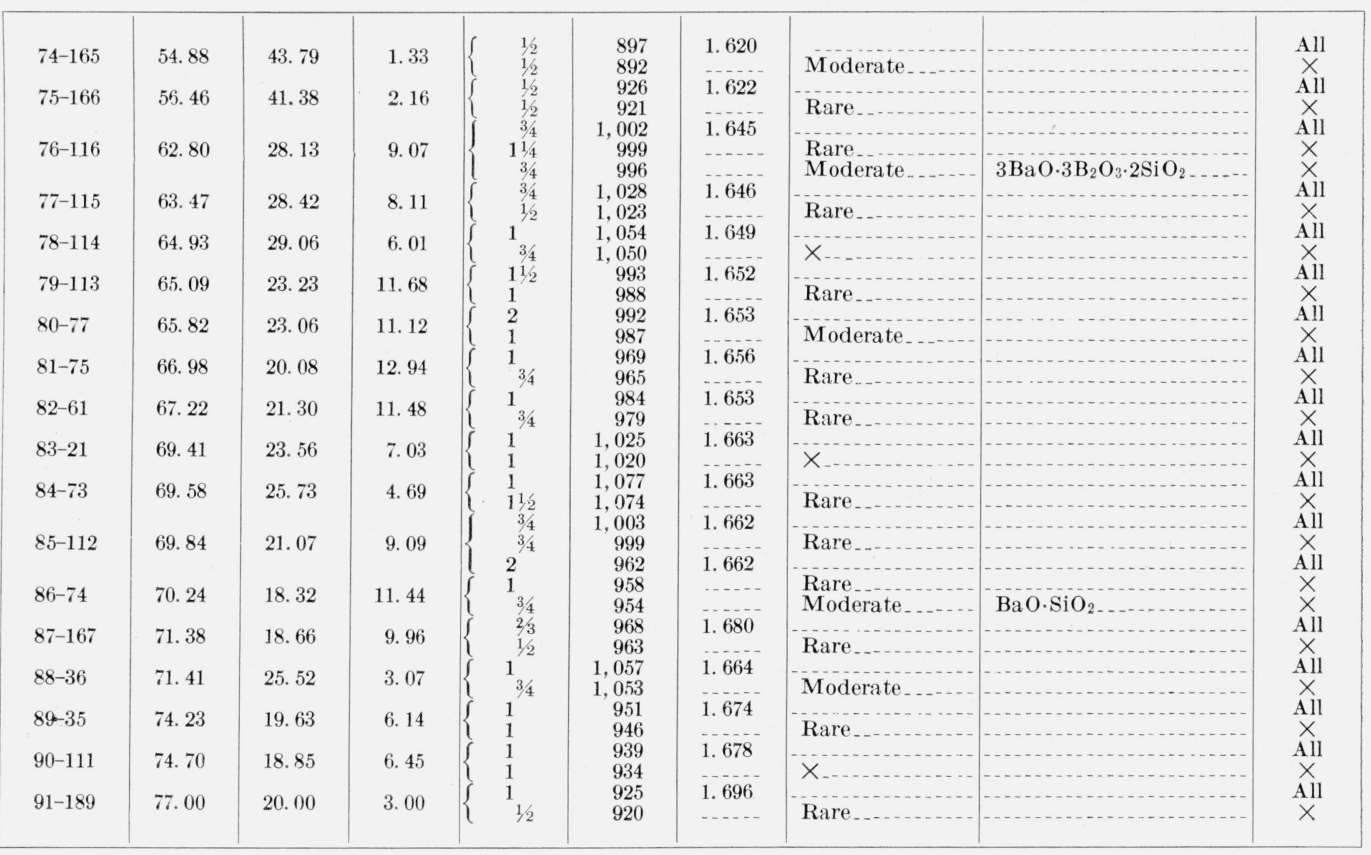

G. Compositions in the $\mathrm{BaO} \cdot 2 \mathrm{~B}_{2} \mathrm{O}_{3}$ primary field

\begin{tabular}{|c|c|c|c|c|c|c|c|c|c|}
\hline & & & & $1 / 2$ & 845 & 1. 568 & & & All \\
\hline $92-96$ & 39.30 & 55. 04 & 5. 66 & $\begin{array}{l}1 / 2 \\
1 / 2\end{array}$ & $\begin{array}{l}841 \\
835\end{array}$ & & $\begin{array}{l}\text { Rare } \\
\text { Moderate. }\end{array}$ & $\mathrm{BaO} \cdot 4 \mathrm{~B}_{2} \mathrm{O}_{3}$ & $\stackrel{x}{x}$ \\
\hline $93-147$ & 40.26 & 52.80 & 6. 94 & $1 / 2$ & $\begin{array}{l}841 \\
839\end{array}$ & 1. 573 & Rare & & $\underset{x}{A 11}$ \\
\hline 94-119 & 40.57 & 55.81 & 3. 62 & $1^{1 / 4}$ & $\begin{array}{l}858 \\
853\end{array}$ & 1. 572 & Rare & & All \\
\hline 95-151 & 42.42 & 55.09 & 2. 49 & $1^{1 / 2}$ & $\begin{array}{l}877 \\
872\end{array}$ & 1.578 & Moderate & & All \\
\hline $96-148$ & 42.65 & 47. 25 & 10.10 & $1 / 1$ & $\begin{array}{l}841 \\
838\end{array}$ & 1. 588 & Rare & & All \\
\hline $97-149$ & 43.49 & 45. 30 & 11. 21 & $1 / 1$ & 840 & 1. 589 & Bare & & All \\
\hline $98-120$ & 44. 21 & 49.81 & 5. 98 & $1 / 2$ & $\begin{array}{l}870 \\
867\end{array}$ & 1. 592 & Bare & & All \\
\hline $99-162$ & 46. 25 & 46.44 & 7.31 & $1 / 2$ & 885 & 1. 595 & Bare & & Âll \\
\hline $100-152$ & 48.05 & 47. 56 & 4. 39 & 3 & 884 & 1. 598 & Bor & & All \\
\hline $101-203$ & 48.55 & 46.90 & 4. 55 & 1 & $\begin{array}{l}801 \\
884 \\
877\end{array}$ & 1.602 & Moderate & & All \\
\hline $102-164$ & 52.90 & 45. 71 & 1. 39 & $\begin{array}{l}1 / 2 \\
1\end{array}$ & $\begin{array}{l}906 \\
900\end{array}$ & 1.617 & Rare & & $\underset{x}{\text { All }}$ \\
\hline $103-80$ & 53.69 & 44. 37 & 1.94 & $1^{1 / 2}$ & $\begin{array}{l}897 \\
894\end{array}$ & 1.618 & Rare & & $\begin{array}{l}A 1 \\
\times 1\end{array}$ \\
\hline
\end{tabular}

See footnotes at end of table. 
TABLE 3. Data on liquidus determinations in the system $\mathrm{BaO}-\mathrm{B}_{2} \mathrm{O}_{3}-\mathrm{SiO}_{2}-$-Continued

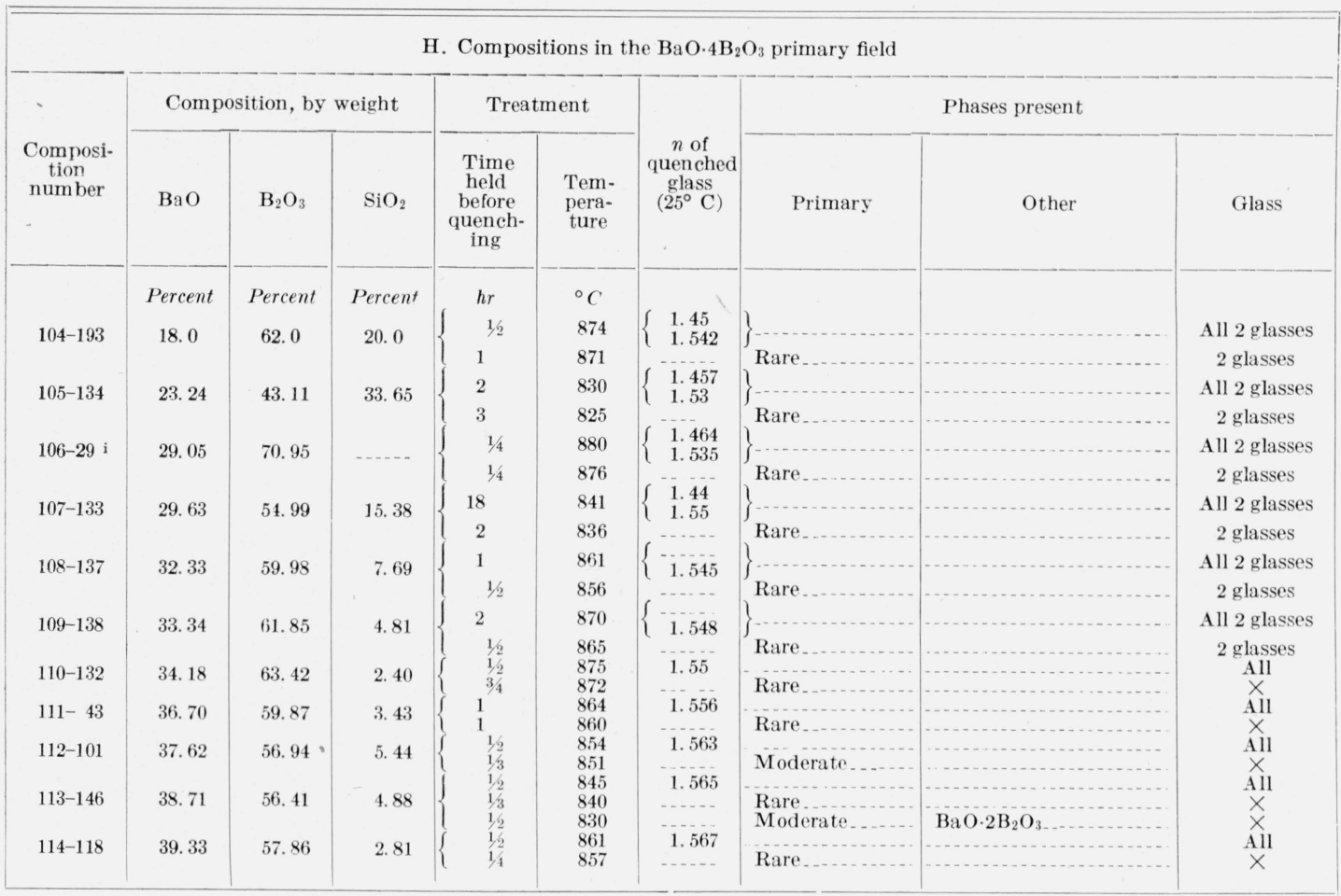

I. Compositions in the $\mathrm{SiO}_{2}{ }^{\mathrm{j}}$ primary field

\begin{tabular}{|c|c|c|c|c|c|c|c|c|c|}
\hline $115-136$ & 10.10 & 18. 75 & 71.15 & 2 & е 960 & $\{1.459$ & $\times$ & & 2 Glasses \\
\hline $116-135$ & 17.51 & 32.49 & 50.00 & $\left\{\begin{array}{l}17 \\
11 / 2\end{array}\right.$ & $\begin{array}{l}830 \\
821\end{array}$ & $\left\{\begin{array}{l}\sim 1.45 \\
\sim 1.55\end{array}\right.$ & & & $\begin{array}{c}\text { All } 2 \text { glasses } \\
2 \text { Glasses }\end{array}$ \\
\hline $117-195$ & 20.0 & 30.0 & 50.0 & $1 / 2$ & e 860 & $\{\sim 1.56$ & & & 2 Glasses \\
\hline $118-194$ & 20.0 & 40.0 & 40.0 & $\left\{\begin{array}{l}1 / 2 \\
1 / 2\end{array}\right.$ & $\begin{array}{r}\text { e } 826 \\
821\end{array}$ & $\left\{\begin{array}{r}1.56 \\
1.55\end{array}\right.$ & & $\mathrm{BaO} \cdot 4 \mathrm{~B}_{2} \mathrm{O}_{3} \ldots$ & $\begin{array}{l}2 \text { Glasses } \\
2 \text { Glasses }\end{array}$ \\
\hline $119-145$ & 25.55 & 11.41 & 63.04 & $1 / 2$ & e 1,278 & $\left\{\begin{array}{l}\sim 1.47 \\
\sim 1.57\end{array}\right.$ & & . & 2 Glasses \\
\hline $120-142$ & 29.85 & 27.18 & 42.97 & $\left\{\begin{array}{r}18 \\
2\end{array}\right.$ & $\begin{array}{r}1,018 \\
960\end{array}$ & $\left\{\begin{array}{l}\sim 1.46 \\
\sim 1.58\end{array}\right.$ & & & $\begin{array}{l}\text { All } 2 \text { glasses } \\
2 \text { Glasses }\end{array}$ \\
\hline $121-144$ & 34.13 & 15. 25 & 50.62 & $\left\{\begin{array}{l}1 / 2 \\
1 / 2\end{array}\right.$ & $\begin{array}{l}1,350 \\
1,314\end{array}$ & $\left\{\begin{array}{l}\sim 1.46 \\
\sim 1.56\end{array}\right.$ & & & $\begin{array}{l}\text { All } 2 \text { glasses } \\
2 \text { Glasses }\end{array}$ \\
\hline $122-161$ & 37.76 & 16.87 & 45.37 & 1 & e 1,201 & $\{\sim 1.57$ & & & 2 Glasses \\
\hline $123-141$ & 38.54 & 35.09 & 26.37 & $\left\{\begin{array}{r}1 \\
56\end{array}\right.$ & $\begin{array}{r}\text { e } 922 \\
910\end{array}$ & $\left\{\begin{array}{c}\sim 1,46 \\
\hdashline \cdots\end{array}\right.$ & & & $\begin{array}{l}2 \text { Glasses } \\
2 \text { Glasses }\end{array}$ \\
\hline $124-140$ & 42. 63 & 38.82 & 18.55 & $\left\{\begin{array}{l}1 \\
2\end{array}\right.$ & $\begin{array}{l}915 \\
910\end{array}$ & $\{\sim 1.59$ & & & All 2 glasses \\
\hline $125-27$ & 39.28 & 51.91 & 8.81 & $\begin{array}{l}3 \\
11 / 2 \\
11 / 2\end{array}$ & $\begin{array}{l}910 \\
826 \\
823 \\
814\end{array}$ & 1.573 & (n) & $\mathrm{BaO} \cdot 2 \mathrm{~B}_{2} \mathrm{O}_{3}$ & $\begin{array}{l}\text { All } \\
\times \\
\times\end{array}$ \\
\hline $126-102$ & 40.36 & 48.52 & 11.12 & $1^{1 / 3}$ & $\begin{array}{l}838 \\
830 \\
816\end{array}$ & 1.577 & (n) & $\mathrm{BaO} 2 \mathrm{~B}_{2} \mathrm{O}_{3}$ & $\begin{array}{l}\text { All } \\
\times \\
\times\end{array}$ \\
\hline $127-44$ & 41.37 & 45. 43 & 13.20 & $\begin{array}{r}1 \\
1 \\
16\end{array}$ & $\begin{array}{l}834 \\
830 \\
810\end{array}$ & $\begin{array}{r}1.582 \\
-1 .\end{array}$ & Rare & & \\
\hline $128-160$ & 41. 72 & 18. 64 & 39.64 & $\begin{array}{r}16 \\
1 \\
1 / 6\end{array}$ & $\begin{array}{r}810 \\
\text { e } 1,200 \\
860\end{array}$ & 1.588 & X & $\mathrm{BaO} \cdot 2 \mathrm{~B}_{2} \mathrm{O}_{3 \ldots}$ & $\stackrel{x}{x}$ \\
\hline $129-103$ & 41.81 & 44.02 & 14.17 & $\left\{\begin{array}{l}1 / 2 \\
1 / 2 \\
1 / 2 \\
1 / 4\end{array}\right.$ & $\begin{array}{l}850 \\
835 \\
860\end{array}$ & 1........ & (n) & $\mathrm{BaO} \cdot 2 \mathrm{~B}_{2} \mathrm{O}_{3 \ldots}$ & $\stackrel{x}{x}$ \\
\hline $130-104$ & 42.46 & 42.01 & 15.53 & $\begin{array}{l}1 / 4 \\
1 / 4\end{array}$ & $\begin{array}{l}860 \\
847\end{array}$ & 1.588 & (n) & & $\stackrel{A}{x}$ \\
\hline $131-139$ & 43. 91 & 39.98 & 16.11 & $2^{1 / 2}$ & $\begin{array}{l}891 \\
880 \\
870\end{array}$ & 1.592 & X & $3 \mathrm{BaO} \cdot 3 \mathrm{~B}_{2} \mathrm{O}_{3} \cdot 2 \mathrm{SiO}_{2}$ & $\stackrel{\text { All }}{x}$ \\
\hline $132-131$ & 45.05 & 20.12 & 34.83 & $\begin{array}{l}1 \\
1 \\
1\end{array}$ & $\begin{array}{r}1,102 \\
1,055 \\
955\end{array}$ & $\begin{array}{c}1.595 \\
1.600\end{array}$ & Rare & (1) & $\stackrel{\text { All }}{\times}$ \\
\hline $133-45$ & 47. 69 & $\begin{array}{l}32.52 \\
25.92\end{array}$ & 26.39 & $\begin{array}{l}1 \\
11 / 2 \\
1 \\
1 / 2\end{array}$ & $\begin{array}{r}950 \\
900 \\
1,101 \\
996\end{array}$ & 1.606 & $\begin{array}{l}\text { Rare } \\
\times\end{array}$ & $3 \mathrm{BaO} \cdot 3 \mathrm{~B}_{2} \mathrm{O}_{3} \cdot 2 \mathrm{SiO}_{2}$ & $\begin{array}{l}\text { All } \\
x \\
x \\
\text { All } \\
x\end{array}$ \\
\hline $135-105$ & 48. 79 & 22.49 & 28.72 & $\begin{array}{l}11 / 2 \\
1\end{array}$ & $\begin{array}{l}1,008 \\
1,004\end{array}$ & 1. 610 & $\times$ & (1) & $\stackrel{A}{x ! 1}$ \\
\hline
\end{tabular}

See footnotes at end of table. 
TABLE 3. Data on liquidus determinations in the system $\mathrm{BaO}-\mathrm{B}_{2} \mathrm{O}_{3}-\mathrm{SiO}_{2}$-Continued

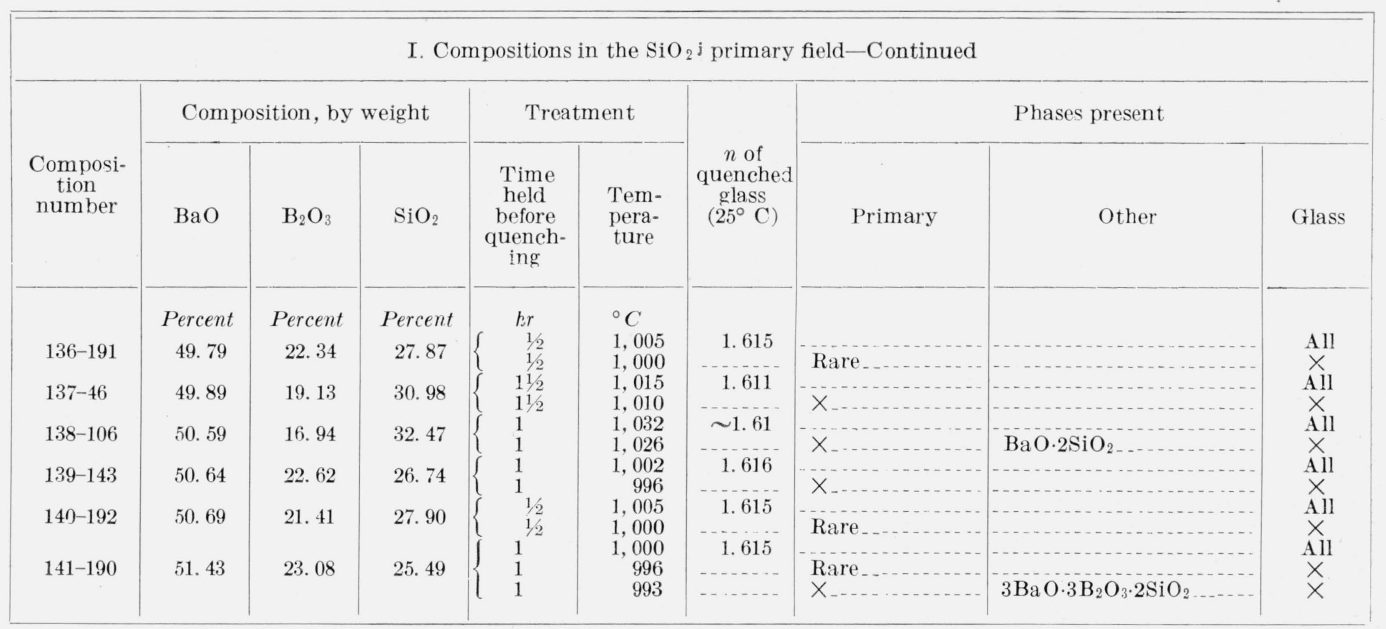

J. Compositions in the $3 \mathrm{BaO} \cdot 3 \mathrm{~B}_{2} \mathrm{O}_{3} \cdot 2 \mathrm{SiO}_{2}$ primary field

\begin{tabular}{|c|c|c|c|c|c|c|c|c|c|}
\hline $142-66$ & 44. 98 & 41.87 & 13.15 & 1 & $\begin{array}{l}850 \\
844\end{array}$ & 1.593 & & & All \\
\hline $143-97$ & 45.80 & 39.94 & 14.26 & 1 & $\begin{array}{l}8+4 \\
897\end{array}$ & 1. 601 & & & All \\
\hline $145-96$ & 45.80 & 39.94 & 14.20 & $1_{1 / 6}$ & $\begin{array}{l}892 \\
904\end{array}$ & 600 & Rare..... & & $\stackrel{x}{1}$ \\
\hline $144-163$ & 47.43 & 44.49 & 8.08 & $1^{1 / 2}$ & $\begin{array}{l}904 \\
901\end{array}$ & & Rare & - n & $x$ \\
\hline $145-65$ & 47. 92 & 35.05 & 17.03 & $\begin{array}{l}1 \\
1\end{array}$ & $\begin{array}{l}965 \\
960\end{array}$ & 1.607 & Rare............ & & $\stackrel{\text { All }}{x}$ \\
\hline $146-121$ & 48.49 & 42.74 & 8.77 & $\begin{array}{l}1 \\
1\end{array}$ & $\begin{array}{l}924 \\
919\end{array}$ & 1. 604 & & & $\widehat{A l l}$ \\
\hline $147-122$ & 49.02 & 41.86 & 9.12 & $\begin{array}{l}1 \\
1\end{array}$ & $\begin{array}{l}936 \\
932\end{array}$ & 1. 605 & & & All \\
\hline $148-204$ & 49.25 & 45. 96 & 4. 79 & $\begin{array}{l}1 / 2 \\
1 / 2\end{array}$ & $\begin{array}{l}890 \\
886\end{array}$ & 1. 604 & Moderate & Vervare $\mathrm{Bg} \cdot 2 \mathrm{~B} \mathrm{O}_{3}$ & All \\
\hline $149-205$ & 49.95 & 45.02 & 5.03 & $\begin{array}{l}1 / 2 \\
1 / 6\end{array}$ & $\begin{array}{l}904 \\
899\end{array}$ & 1. 606 & & 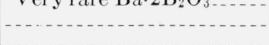 & All \\
\hline $150-67$ & 50.85 & 28.24 & 20.91 & $1^{\prime 2}$ & $\begin{array}{l}986 \\
980\end{array}$ & 1. 613 & nare & & All \\
\hline $151-198$ & 52.06 & 21.14 & 26.80 & 1 & $\begin{array}{l}980 \\
995 \\
989\end{array}$ & 1. 617 & & (n) & All \\
\hline $152-98$ & 52.09 & 25.33 & 22.58 & $1 / 2$ & 997 & 1. 623 & -... & $\cdots$ & All \\
\hline $153-123$ & 52,10 & 36.78 & 11.12 & $\begin{array}{l}1 / 2 \\
1 / 4\end{array}$ & $\begin{array}{l}992 \\
985\end{array}$ & 1. 617 & & & All \\
\hline & & 30.18 & 11.12 & $\begin{array}{r}1 / 4 \\
11 / 2\end{array}$ & $\begin{array}{l}979 \\
900\end{array}$ & 1.615 & Rare..... & n. & $\stackrel{\times}{\times}$ \\
\hline $154-38$ & 53.41 & 43. 90 & 2. 69 & 1 & 894 & (..... & Rare & & $\cdot x$ \\
\hline & & & & $\begin{array}{l}2 \\
1\end{array}$ & $\begin{array}{l}886 \\
998\end{array}$ & 620 & Moderate. & $\mathrm{BaO} \cdot 2 \mathrm{~B}_{2} \mathrm{O}_{3-}$ & x \\
\hline $155-99$ & 53.56 & 21.92 & 24.52 & 1 & 995 & & Rare .............. & (1) & $\times$ \\
\hline $156-92$ & 53.66 & 30.78 & 15.56 & $\begin{array}{l}1 / 3 \\
1 / 4\end{array}$ & $\begin{array}{r}1,003 \\
998\end{array}$ & 1. 622 & $x$ & & $\stackrel{\text { All }}{x}$ \\
\hline $157-50$ & 54.11 & 27.18 & 18.71 & 1 & $\begin{array}{r}1,000 \\
996\end{array}$ & 1. 622 & Med & & All \\
\hline $158-34$ & 54,32 & 24.37 & 21,31 & $11 / 2$ & 1,001 & 1. 625 & Nod & & All \\
\hline & & & & $1^{11 / 2}$ & 998 & 1600 & Moderate.. & - & $\times$ \\
\hline 159-39 & 54.38 & 39.45 & 6.17 & 11,4 & 954 & 1.020 & Rare & & A11 \\
\hline $160-93$ & 54.46 & 20.67 & 24.87 & $\begin{array}{l}1 \\
1\end{array}$ & $\begin{array}{l}999 \\
995\end{array}$ & 1. 622 & Rare & & $\underset{x}{\text { All }}$ \\
\hline & & & & $11 / 2$ & 984 & 109 & Moderate....... & $\mathrm{BaO} \cdot 2 \mathrm{SiO}_{2} \ldots$ & $\hat{x}$ \\
\hline $161-24$ & 55.24 & 35.50 & 9. 26 & $1^{1 / 2}$ & $\begin{array}{l}988 \\
981\end{array}$ & 1.628 & Rare............. & (n) & $\stackrel{\text { All }}{x}$ \\
\hline $162-87$ & 55.72 & 32.52 & 11. 76 & $\begin{array}{l}1 / 4 \\
1 / 2\end{array}$ & $\begin{array}{r}1,002 \\
998\end{array}$ & 1. 628 & Rare & & All \\
\hline $163-40$ & 56.56 & 29.47 & 13.97 & $\begin{array}{l}1 \\
1\end{array}$ & $\begin{array}{l}1,005 \\
1,000\end{array}$ & 1. 629 & Moderate & & All \\
\hline $164-68$ & 57.15 & 26. 79 & 16.06 & $\begin{array}{l}11 / 2 \\
11 / 6\end{array}$ & $\begin{array}{l}1,008 \\
1,005\end{array}$ & 1. 631 & Moderate & & $\widehat{A l l}$ \\
\hline $165-32$ & 57.67 & 24.37 & 17. 96 & $11 / 2$ & 1,008 & 1.633 & mouera & & All \\
\hline & & & & 1 & $\begin{array}{r}1,004 \\
985\end{array}$ & 1. 631 & D & & $\stackrel{x}{\text { All }}$ \\
\hline $166-57$ & 57.72 & 34.85 & 7.43 & $1^{3 / 4}$ & $\begin{array}{l}980 \\
951\end{array}$ & (n) & $\begin{array}{l}\text { Rare } \\
\text { Moderate............. }\end{array}$ & & $\stackrel{x}{x}$ \\
\hline & & & & $\begin{array}{l}1 \\
1\end{array}$ & $\begin{array}{r}940 \\
1,005\end{array}$ & 1.634 & Large ........... & $\mathrm{BaO} \cdot \mathrm{B}_{2} \mathrm{O}_{3} \ldots \ldots$ & $\stackrel{x}{x}$ \\
\hline $167-25$ & 57.83 & 23.66 & 18.51 & 1 & 1,001 & & Rare & & Ain \\
\hline $168-88$ & 58.11 & 21.83 & 20.06 & $\begin{array}{l}2 \\
2\end{array}$ & $\begin{array}{l}1,005 \\
1,000\end{array}$ & 1. 631 & $\times$ & & $\stackrel{\text { All }}{x}$ \\
\hline $169-69 \mathrm{k}$ & 58.30 & 26.48 & 15.22 & $\begin{array}{l}1 / 2 \\
1 / 6\end{array}$ & $\begin{array}{l}1,013 \\
1,011\end{array}$ & 1. 638 & Vervrare & & $\widehat{A l l}$ \\
\hline $170-89$ & 58.63 & 19.51 & 21.86 & $21 / 2$ & $\begin{array}{r}1,002 \\
998\end{array}$ & 1. 634 & Rare & & $\widehat{A} \times$ \\
\hline $171-174$ & 59.82 & 18.43 & 21.75 & 1 & $\begin{array}{l}997 \\
994\end{array}$ & 1. 638 & $x$ & & All \\
\hline $172-197$ & 61.30 & 19.49 & 19. 21 & $\begin{array}{l}1 \\
1\end{array}$ & $\begin{array}{l}995 \\
990\end{array}$ & 1. 641 & Rare & & $\widehat{A} \times$ \\
\hline & & & & 1 & 1,007 & 1. 642 & & - & $\widehat{A l l}$ \\
\hline $173-117$ & 61.41 & 27.52 & 11.07 & $\begin{array}{l}1 \\
1\end{array}$ & $\begin{array}{r}1,005 \\
994\end{array}$ & (n.... & $\begin{array}{l}\text { Rare ............ } \\
\text { Moderate........ }\end{array}$ & $\mathrm{BaO} \cdot \mathrm{B}_{2} \mathrm{O}_{3}$ & $\stackrel{x}{x}$ \\
\hline
\end{tabular}

See footnotes at end of table. 
TABI.E 3. Data on liquidus determinations in the system $\mathrm{BaO}-\mathrm{B}_{2} \mathrm{O}_{3}-\mathrm{SiO}_{2}$ - Continued

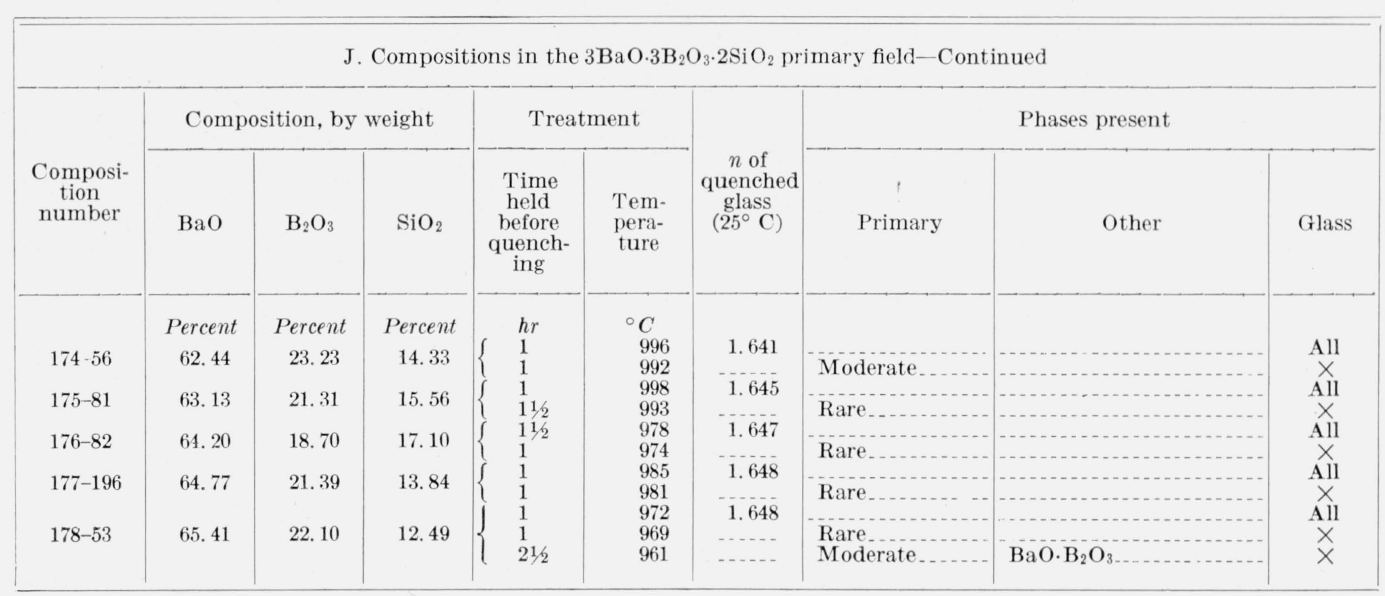

a X-ray powder pattern of crystals grown at $1,100^{\circ} \mathrm{C}$ showed very slight irregular shift in $d$ spacings from pure $\mathrm{BaO} \cdot 2 \mathrm{SiO}_{2}$. No detectable change in optical shift in $d$
properties.

b Solid solution with $\mathrm{BaO} \cdot 2 \mathrm{SiO}_{2}$ and with barium borates, confirmed by optical and $\mathrm{X}$-ray data.

'X-ray powder pattern of primary phese erystals showed a maximum shift of $0.02 \mathrm{~A}$ in $d$ spacings from pure $2 \mathrm{BaO} \cdot 3 \mathrm{SiO}_{2}$. No detectable change in optical properties.

d Includes quench growths and barium platinates.

features of the $\mathrm{BaO}-\mathrm{SiO}_{2}$ system. Melting points, optical properties, and X-ray diffraction data for all compounds in the system were determined; and a microscopic and X-ray study of the $\mathrm{BaO} \cdot 2 \mathrm{SiO}_{2}-$ $2 \mathrm{BaO} \cdot 3 \mathrm{SiO}_{2}$ solid solution area was made.

The melting points and optical properties of the compounds were found to be, in general, in close agreement with the reported values $[15,16]$. Eskola [15] reported the melting point of dibarium silicate $\left(2 \mathrm{BaO} \cdot \mathrm{SiO}_{2}\right)$ as being above that of platinum. $\mathrm{By}$ a method of optical pyrometry [19], it was possible to estimate the melting point as $1,815 \pm 25^{\circ} \mathrm{C}$. Microscopic examination of $2 \mathrm{BaO} \cdot \mathrm{SiO}_{2}$ that had been heated above $1,800^{\circ} \mathrm{C}$ gave some new optical data. The material appeared as irregular grains showing good cleavage parallel to the plane containing the alpha and beta vibration directions; it was biaxial positive with a very small $2 \mathrm{~V}$ ( 5 to $\left.10^{\circ}\right)$; $\alpha=1.800, \beta=1.80+, \gamma=1.826$.

Table 2 gives a comparison of the X-ray powder pattern diffraction data obtained in this study with that of Austin's [20]. Although agreement between the two sets of data is reasonably good, enough differences exist to warrant recording. It can be seen from table 2 that minor differences exist in interplanar spacings and relative intensities between corresponding patterns. The present study reveals additional interplanar spacings for most of the compounds, in particular for $\mathrm{BaO} \cdot 2 \mathrm{SiO}_{2}$. Perhaps the most notable difference in the corresponding pairs of patterns is the lack of agreement of the three major peaks (identified as (1), (2), and (3) in table 2 ). Only the numbers (1) and (2) peaks for $2 \mathrm{BaO} \cdot \mathrm{SiO}_{2}$ and the number (1) peaks for $\mathrm{BaO} \cdot \mathrm{SiO}_{2}$ and $2 \mathrm{BaO} \cdot 3 \mathrm{SiO}_{2}$ agree between the two sets of data. The remainder of the peaks are either interchanged in order of intensity or are entirely different. The discrepancies in order of intensity of the three major
- Liquidus greater than this temperature.

'Temperature of a ternary invariant point.

s All glass devitrified to form quench grow ths

h Glass and quench growths.

To check liquidus of binary immiscibility region.

i Most difficult of the primary phase regions studied and, consequently, the least accurate; tridymite only form identified.

$\mathbf{k}$ Composition of $3 \mathrm{BaO} \cdot 3 \mathrm{~B}_{2} \mathrm{O}_{3} \cdot 2 \mathrm{SiO}_{2}$.

peaks for corresponding sets of data are due probably to orientation. In this study, an attempt was made to minimize orientation in the sample during specimen mounting. The finely ground material (passing a No. 200 mesh screen) was neither packed nor scraped at any stage of preparation but was sprinkled freely over a microscope slide (which served as a specimen holder) that had been smeared with a thin layer of petrolatum.

Study of the $\mathrm{BaO} \cdot \mathrm{SiO}_{2}-2 \mathrm{BaO} \cdot 3 \mathrm{SiO}_{2}$ solid solution area was based on the following four compositions: 100 percent of $\mathrm{BaO} \cdot 2 \mathrm{SiO}_{2}\left(0\right.$ percent of $2 \mathrm{BaO} \cdot 3 \mathrm{SiO}_{2}$ ), 70.8 percent of $\mathrm{BaO} \cdot 2 \mathrm{SiO}_{2}(29.2$ percent of $2 \mathrm{BaO}$. $\left.3 \mathrm{SiO}_{2}\right), 37.5$ percent of $\mathrm{BaO} \cdot 2 \mathrm{SiO}_{2}$ (62.5 percent of $\left.2 \mathrm{BaO} \cdot 3 \mathrm{SiO}_{2}\right)$, and 0 percent of $\mathrm{BaO} \cdot 2 \mathrm{SiO}_{2}$ (100 percent of $2 \mathrm{BaO} \cdot 3 \mathrm{SiO} 2)$. Results of the optical examination agreed with Eskola's study [15]. The X-ray data, however, did not conclusively show a solid solution series. The powder pattern for the composition containing 70.8 percent of $\mathrm{BaO} \cdot \mathrm{SiO}_{2}$ (29.2 percent of $2 \mathrm{BaO} \cdot 3 \mathrm{SiO}_{2}$ ) showed several double peaks which indicated the presence of two phases. Even after an additional melting and grinding of the mixture, these double peaks persisted. It is believed that single crystal work, which would permit the determination of unit cell sizes, would be the most suitable method of studying the solid solution area by means of X-rays.

\section{Experimental Results and Discussion}

\subsection{General Statement}

Table 3 gives the essential quenching data for the 178 compositions studied, grouped according to primary fields of crystallization. The compositions within each primary field are arranged according to increasing percentage of $\mathrm{BaO}$. The locations of the 


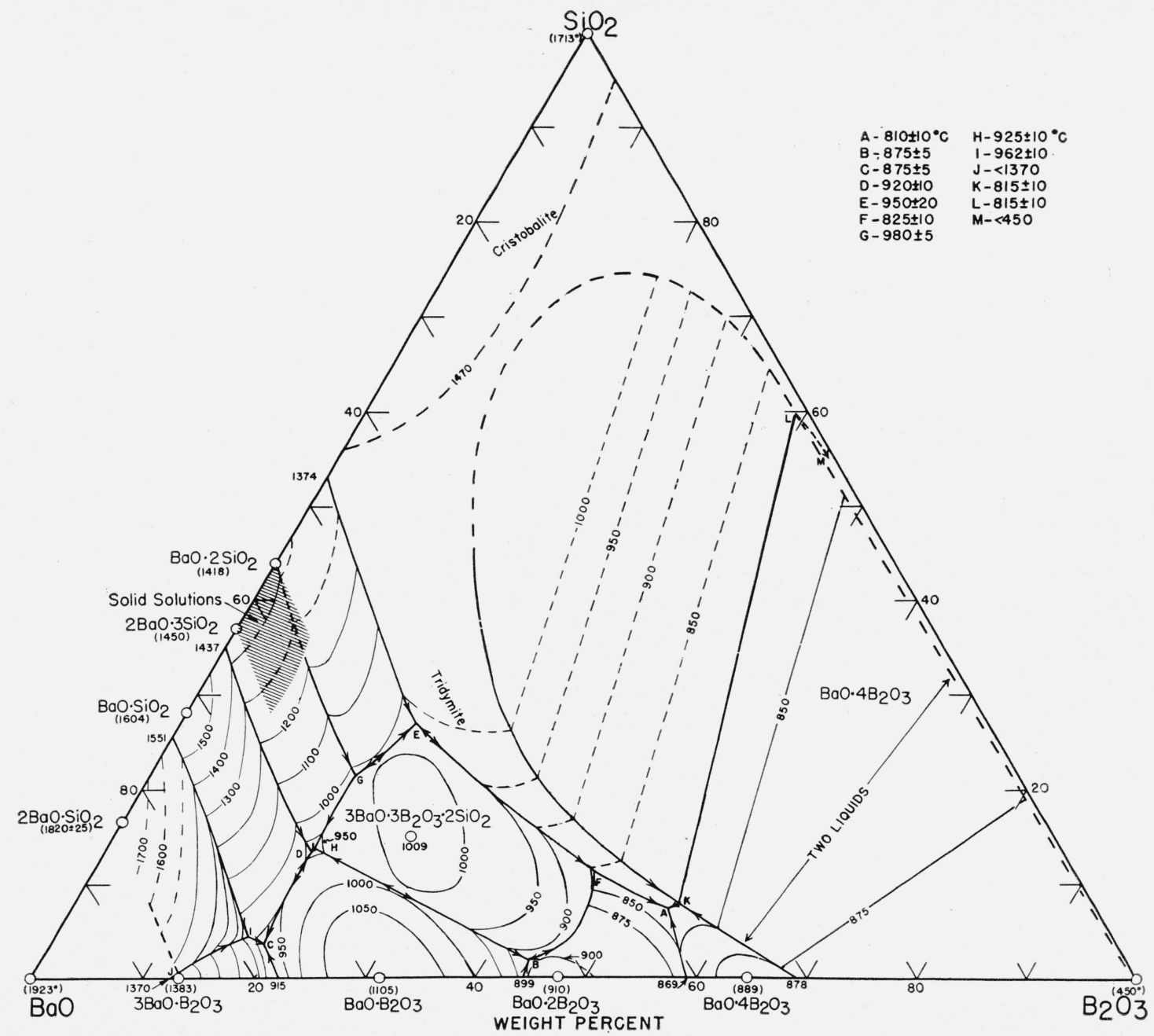

Figure 6. Phase equilibrium diagram for the system $\mathrm{BaO}-\mathrm{B}_{2} \mathrm{O}_{3}-\mathrm{SiO}_{2}$, showing isotherms, boundaries, and invariant points.

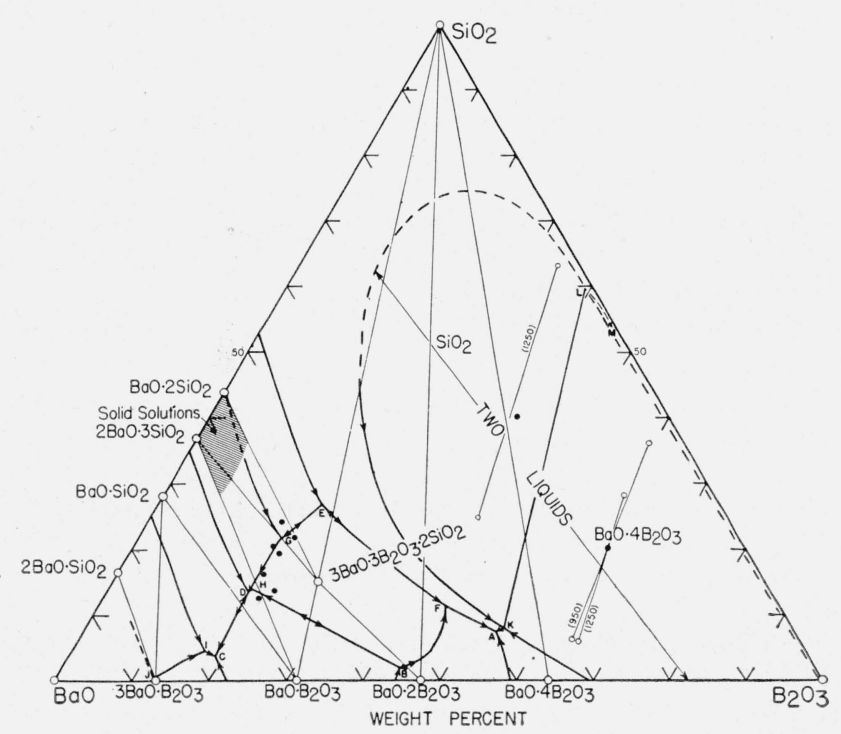

Figure 7. Composition triangles in the system $\mathrm{BaO}-\mathrm{B}_{2} \mathrm{O}_{3}-$ $\mathrm{SiO}_{2}$. compositions studied are shown in figure 1. The data from table 3 are plotted in figures 6 and 7 .

Figure 6 shows the ternary diagram for the system, with the temperatures of the invariant points listed alongside. The heary lines, solid and dashed, represent the phase boundaries and delineate the primary phase areas. The arrows indicate the direction of falling temperature, in the conventional manner. Isotherms are drawn generally at 100-deg intervals, but in some cases at 50- or 25-deg intervals. The temperatures for the polymorphic inversions of silica, such as quartz to tridymite and tridymite to cristobalite, could not be obtained experimentally, but for the case of the tridymite-cristobalite inversion the assumption was made that the inversion temperature is the same as for pure silica, namely, $1,470^{\circ} \mathrm{C}$. Little data could be obtained in the regions very rich in $\mathrm{BaO}$, for example, in the $2 \mathrm{BaO} \cdot \mathrm{SiO}_{2}$ and $\mathrm{BaO}$ primary phase areas, (1) because of the reaction of the samples with platinum at high temperatures to form barium platinates, (2) because of the extremely rapid rate of devitrification of samples during quenching, and (3) because of the rapid hydration of samples 
after quenching. Experimental difficulties due largely, it is believed, to the high viscosity of the melts were encountered, likewise, in studying most of the two-liquid immiscibility region and the silica primary phase area. It should be noted that such factors were encountered as very fine crystals difficult to identify, evidences of nonequilibrium conditions, failure to obtain clear-cut separation of the immiscible liquids, and inability over most of the silica field to determine liquidus temperatures. This portion of the diagram, therefore, is of a tentative nature, but it is thought that the relationships as shown are essentially correct.

Each of the eight binary compounds shows a primary phase region in the ternary diagram (fig. 6). Only one ternary compound, $3 \mathrm{BaO} \cdot 3 \mathrm{~B}_{2} \mathrm{O}_{3} \cdot 2 \mathrm{SiO}_{2}$, is found. The system contains 5 ternary eutectics, lettered A, B, C, D, E; five ternary invariant points not eutectics (reaction points) lettered F, G, H, I, J; and 2 quintuple points, lettered $\mathrm{K}$ and $\mathrm{L}$, at which 2 liquid phases are in equilibrium with 2 crystalline phases and vapor. With the exception of point $J$, temperatures of these invariant points range between $810^{\circ}$ and $980^{\circ} \mathrm{C}$. The invariant point $\mathrm{J}$ is under $1,370^{\circ} \mathrm{C}$, the value of the binary eutectic between $\mathrm{BaO}$ and $3 \mathrm{BaO} \cdot \mathrm{B}_{2} \mathrm{O}_{3}$. However, as point $\mathrm{J}$ is located near the binary eutectic, it is to be expected that its liquidus value is not much lower. One other invariant point, $M$, representing the equilibrium between silica, boric oxide, and $\mathrm{BaO} \cdot 4 \mathrm{~B}_{2} \mathrm{O}_{3}$ is postulated [21] to lie somewhere in the narrow region between the $\mathrm{B}_{2} \mathrm{O}_{3}-\mathrm{SiO}_{2}$ binary and the boundary between the two immiscible liquids and one-liquid regions. The most prominent feature of the diagram is the large extent of the area of two immiscible liquids, which covers approximately 50 percent of the diagram. It is also interesting to note that the $\mathrm{BaO} \cdot 4 \mathrm{~B}_{2} \mathrm{O}_{3}$ and $\mathrm{SiO}_{2}$ primary phase areas include almost 70 percent of the diagram.

Figure 7 shows the eleven composition triangles formed in the system by the Alkemade lines. For the purpose of clarity, isotherms are not shown. Three secondary binary systems are evident: $\mathrm{BaO} \cdot \mathrm{SiO}_{2}-\mathrm{BaO} \cdot \mathrm{B}_{2} \mathrm{O}_{3}, \quad 3 \mathrm{BaO} \cdot 3 \mathrm{~B}_{2} \mathrm{O}_{3} \cdot 2 \mathrm{SiO}_{2}-\mathrm{BaO} \cdot \mathrm{B}_{2} \mathrm{O}_{3}$, and $3 \mathrm{BaO} \cdot 3 \mathrm{~B}_{2} \mathrm{O}_{3} \cdot 2 \mathrm{SiO}_{2}-\mathrm{BaO} \cdot 2 \mathrm{~B}_{2} \mathrm{O}_{3}$ (see also fig. 8 , D, E, F). It cannot be stated with assurance whether or not the system $3 \mathrm{BaO} \cdot 3 \mathrm{~B}_{2} \mathrm{O}_{3} \cdot 2 \mathrm{SiO}_{2}$ $\mathrm{BaO} \cdot 2 \mathrm{SiO}_{2}$ is a binary because the exact nature and extent of the solid solution field is not known (see fig. $8, \mathrm{~A})$. The system $3 \mathrm{BaO} \cdot 3 \mathrm{~B}_{2} \mathrm{O}_{3} \cdot 2 \mathrm{SiO}_{2}-\mathrm{BaO} \cdot \mathrm{B}_{2} \mathrm{O}_{3}$ $\mathrm{BaO} \cdot 2 \mathrm{~B}_{2} \mathrm{O}_{3}$ is seen to be a secondary ternary system. Crystallization paths for compositions in that portion of the baria-borosilicate system where liquid immiscibility does not occur are, in general, straightforward and familiar types. Typical crystallization paths in a two-liquid area are discussed by Flint and Wells [3].

\subsection{Stability Fields of the Binary Compounds}

$$
\text { a. } \mathrm{BaO} \cdot 2 \mathrm{SiO}_{2}
$$

Barium disilicate forms solid solutions with $2 \mathrm{BaO} \cdot 3 \mathrm{SiO}_{2}$ and probably with the barium borates, the partial limits of these solid solutions are shown in figure 6. Compositions studied in the $\mathrm{BaO} \cdot 2 \mathrm{SiO}_{2}$ primary field are given in table 3 , part $\mathbf{A}$. Liquidus temperatures vary from the melting point fof $\mathrm{BaO} \cdot 2 \mathrm{SiO}_{2}\left(1,418^{\circ} \mathrm{C}\right)^{3}$ to a minimum of $950^{\circ} \mathrm{C}$ (point E). Optical properties of the primary phase crystals within the limits of experimental measurements did not differ from those of pure $\mathrm{BaO} \cdot 2 \mathrm{SiO}_{2}$. X-ray diftraction powder pattern data of primary phase crystals grown at $1,100^{\circ} \mathrm{C}$ for two compositions (11 and 13 of table 3 ) showed a very slight and irregular shift of $d$ spacings from the pure $\mathrm{BaO} \cdot 2 \mathrm{SiO}_{2}$. The shift averaged less than $0.01 \mathrm{~A}$, which is about the expected limit of accuracy of the experimental conditions. When liquidus temperatures along the join $\mathrm{BaO} \cdot 2 \mathrm{SiO}_{2}-3 \mathrm{BaO} \cdot 3 \mathrm{~B}_{2} \mathrm{O}_{3} \cdot 2 \mathrm{SiO}_{2}$ are plotted (fig. 8 , A), a distinct and experimentally significant discontinuity is found in the liquidus curve at about $1,250^{\circ} \mathrm{C}$. At this break, the minimum deviation of the curve from one which would show no breaks (that is, a straight line) is $50^{\circ} \mathrm{C}$, a value undoubtedly above the limit of experimental error. It must be concluded, therefore, that the break in the curve is is indicative of a relatively sharp phase change. It would seem likely that the break represents the transition temperature oetween two closely related solid solutio I phases, but the nature of these phases is not known.

\section{b. $2 \mathrm{BaO} \cdot 3 \mathrm{SiO}_{2}$}

Dibarium trisilicate forms solid solutions with $\mathrm{BaO} \cdot 2 \mathrm{SiO}_{2}$ and with the barium borates. The partial limits of the solid solutions are shown in figure 6 . Compositions studied in the $2 \mathrm{BaO} \cdot 3 \mathrm{SiO}_{2}$ primary field are given in table 3, B.

Primary phase crystals from compositions 21, 22, $29,30,33,37$ (table 3, B) gave distinct evidence of being solid solution crystals, both by optical and X-ray methods. No detectable differences in optical properties from pure $2 \mathrm{BaO} \cdot 3 \mathrm{SiO}_{2}$ were found for the primary phase crystals obtained from the remaining compositions. However, crystals from the compositions $25,26,38$, and 42 showed small and irregular shifts in $d$ spacings, to a maximum extent of $0.02 \mathrm{~A}$. $\mathrm{X}$-ray data were not obtained for all compositions in the field, but it seems from the limited data available that all or most of the compositions would show similar slight differences.

When the liquidus temperatures are plotted for the join $3 \mathrm{BaO} \cdot 3 \mathrm{~B}_{2} \mathrm{O}_{3} \cdot 2 \mathrm{SiO}_{2}-2 \mathrm{BaO} \cdot 3 \mathrm{SiO}_{2}$, a discontinuity is found in the liquidus curve at about $1,250^{\circ} \mathrm{C}$ (fig. 8, B), or the same temperature as the break in the $3 \mathrm{BaO} \cdot 3 \mathrm{~B}_{2} \mathrm{O}_{3} \cdot 2 \mathrm{SiO}_{2}-\mathrm{BaO} \cdot 2 \mathrm{SiO}_{2}$ join. The liquidus curve for the join $\mathrm{BaO} \cdot \mathrm{B}_{2} \mathrm{O}_{3}-2 \mathrm{BaO} \cdot 3 \mathrm{SiO}_{2}$ (fig. $8, \mathrm{C}$ ) similarly shows a break in the $2 \mathrm{BaO} \cdot 3 \mathrm{SiO}_{2}$ solid solution field, but it is not as pronounced and occurs at a lower temperature (approximately $1,125^{\circ}$ C). The latter join is more nearly parallel to the isotherms than the other two joins mentioned, and, consequently, the location of the break may be less accurate.

3 Temperature values on the $\mathrm{BaO}-\mathrm{SiO}_{2}$ binary have not been converted to the International Temperature Scale of 1948. 

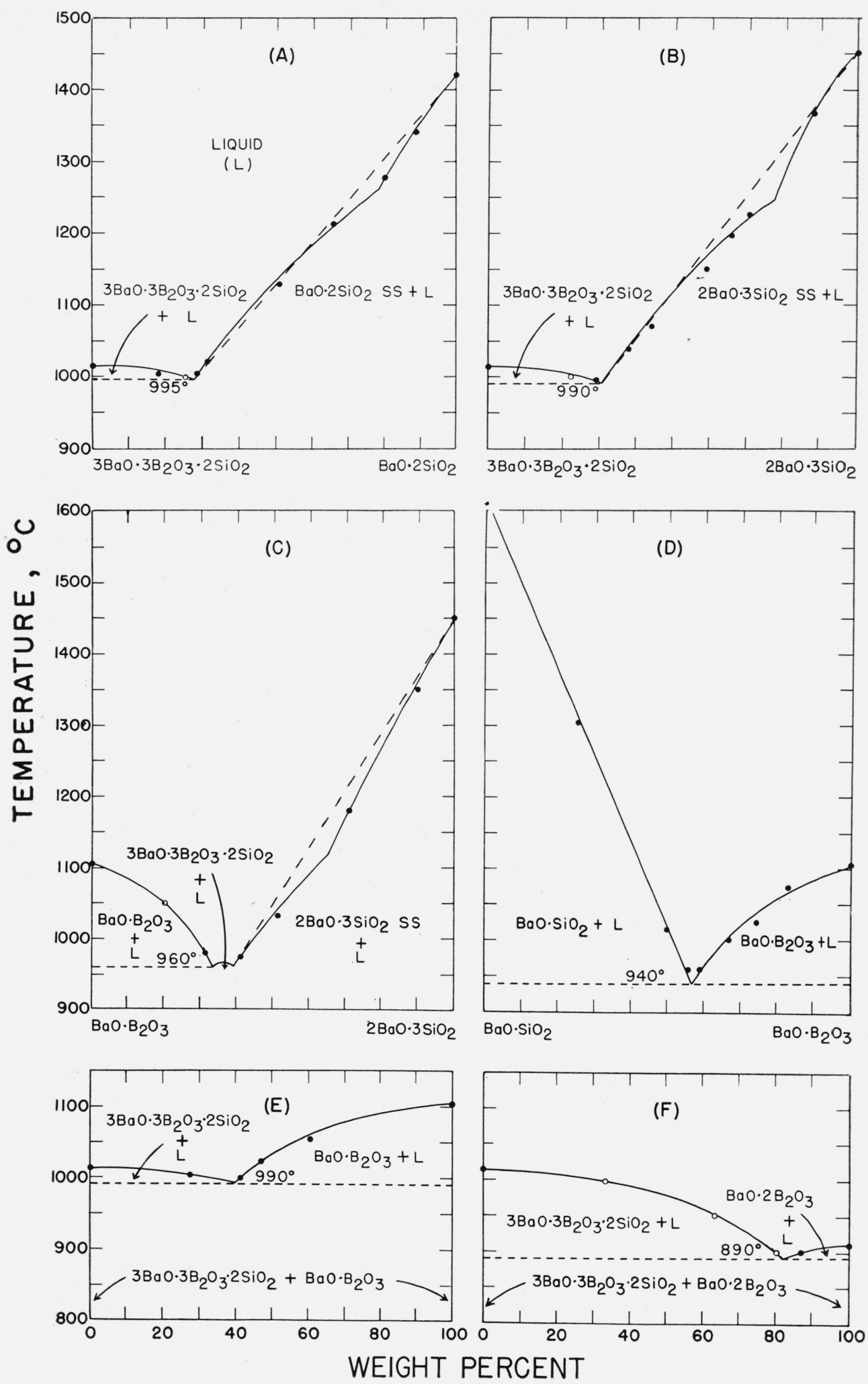

Figure 8. Some binary and pseudo binary systems investigated in the system $\mathrm{BaO}-\mathrm{B}_{2} \mathrm{O}_{3}-\mathrm{SiO}_{2}$ - Actual composition; $\bigcirc$, extrapolation from isotherms; ss, solid solution. 
In his study of the system $\mathrm{BaO}-\mathrm{Al}_{2} \mathrm{O}_{3}-\mathrm{SiO}_{2}$, Thomas [16] found varying indices of refraction for all compositions within the field surrounding the composition $2 \mathrm{BaO} \cdot 3 \mathrm{SiO}_{2}$; and, therefore, he proposed that $2 \mathrm{BaO} \cdot 3 \mathrm{SiO}_{2}$ is not a true compound but merely a particular composition at or near the maximum in a solid solution series. X-ray powder pattern data appeared to confirm his conclusion, although the variations in $d$ values were not as pronounced as the variations in indices of refraction. In the present study, the reverse situation is true, namely, that $\mathrm{X}$-ray measurements in many instances appear to show very slight differences that are not revealed by optical methods. The present study, likewise, does not conclusively prove the existence of a $2 \mathrm{BaO} \cdot 3 \mathrm{SiO}_{2}$ primary phase field of unvarying composition and properties.

The question as to whether or not dibarium trisilicate is a compound becomes especially important when drawing the composition triangle (fig. 7). Obviously, if dibarium trisilicate is not a compound, no Alkemade line can originate from its composition point. Finally, the method of eutectic matrix composition $[22,23]$ was tried.

Seven pertinent ternary compositions, shown by the dots in figure 7 , were selected for study. The formulated compositions were melted and stirred above the liquidus in order to obtain homogeneity. The melts were then crystallized by subjecting them to a slow cooling schedule. The phases in the devitrified samples were identified by petrographic examination.

Most of the samples contained varving amounts of quench growths and small amounts of glass, indicating that equilibrium conditions and complete devitrification were not achieved. In most cases only two crystalline phases were identified. This may be explained on the basis that devitrification of the solid solution crystals consumed all the available liquid before a third phase crystallized. In the case of one composition ( $a$, table 4) for which no solidsolution phase appeared, a different explanation is necessary, as, for example, too small a percentage of fine-grained material intermixed with the quench grow ths of another phase.

Figure 9 and table 4 show the location of the compositions studied, together with the phases identified. Compositions $b$, $c$, and $e$ after devitrification showed three phases: $3 \mathrm{BaO} \cdot 3 \mathrm{~B}_{2} \mathrm{O}_{3} \cdot 2 \mathrm{SiO}_{2}, \mathrm{BaO} \cdot \mathrm{B}_{2} \mathrm{O}_{3}$, and a solid solution with optical properties closely resembling $2 \mathrm{BaO} \cdot 3 \mathrm{SiO}_{2}$. Only $3 \mathrm{BaO} \cdot 3 \mathrm{~B}_{2} \mathrm{O}_{3} \cdot 2 \mathrm{SiO}_{2}$ and $\mathrm{BaO} \cdot \mathrm{B}_{2} \mathrm{O}_{3}$ were found in composition $a$. The data from the four mentioned compositions are consistent with the compatability triangles as drawn. If the correct Alkemade line were really the join $3 \mathrm{BaO} \cdot 3 \mathrm{~B}_{2} \mathrm{O}_{3} \cdot 2 \mathrm{SiO}_{2}-\mathrm{BaO} \cdot \mathrm{SiO}_{2}, \quad$ a possibility if $2 \mathrm{BaO} \cdot 3 \mathrm{SiO}_{2}$ is only a composition in a partial solid solution series between $\mathrm{BaO} \cdot 2 \mathrm{SiO}_{2}$ and $\mathrm{BaO} \cdot \mathrm{SiO}_{2}$, a $\mathrm{BaO} \cdot \mathrm{B}_{2} \mathrm{O}_{3}$ phase should not have been obtained for compositions $b$ and $e$, but rather some indication of $\mathrm{BaO} \cdot \mathrm{SiO}_{2}$. Furthermore, composition $g$ gave no indication of a $3 \mathrm{BaO} \cdot 3 \mathrm{~B}_{2} \mathrm{O}_{3} \cdot 2 \mathrm{SiO}_{2}$ phase whereas $a$, only a few percent away, showed a moderate amount of $3 \mathrm{BaO} \cdot 3 \mathrm{~B}_{2} \mathrm{O}_{3} \cdot 2 \mathrm{SiO}_{2}$. It seems reasonable to suppose that an Alkemade line, as drawn, passes between the two compositions. Compositions $b$ and $e$ both showed $\mathrm{BaO} \cdot \mathrm{B}_{2} \mathrm{O}_{3}$, whereas compositions $d$ and $f$, nearby, did not. The only join that satisfies this result connects $3 \mathrm{BaO} \cdot 3 \mathrm{~B}_{2} \mathrm{O}_{3} \cdot 2 \mathrm{SiO}_{2}$ and $2 \mathrm{BaO} \cdot 3 \mathrm{SiO}_{2}$. Because the composition $2 \mathrm{BaO} \cdot 3 \mathrm{SiO}_{2}$ seems to represent the only logical point for the origination of two Alkemade lines, it must be concluded that $2 \mathrm{BaO} \cdot 3 \mathrm{SiO}_{2}$ is a compound, regardless of the extent of the solid-solution region.

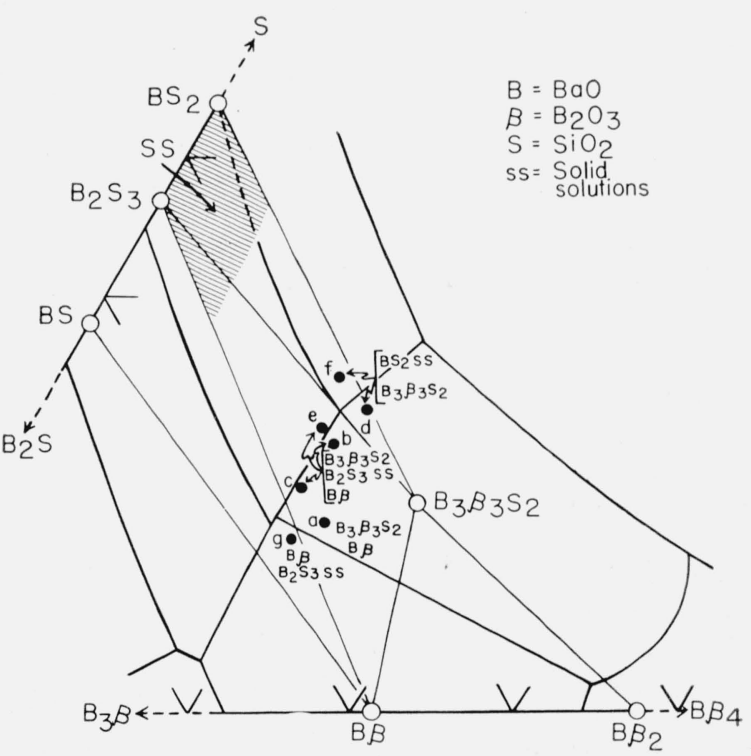

FIGURE 9. Location of $y$ glass compositions and phases identified in the devitrified glasses.

TABLE 4. Phases identified in seven devitrified glasses, in an attempt to determine by the method of eutectic matrix composition whether $2 \mathrm{BaO} \cdot 3 \mathrm{SiO}_{2}$ is a compound or merely a particular composition

\begin{tabular}{|c|c|c|c|c|c|c|c|c|}
\hline \multirow{2}{*}{$\begin{array}{l}\text { Desig- } \\
\text { nation }\end{array}$} & \multirow{2}{*}{$\begin{array}{c}\text { Composi- } \\
\text { tion num- } \\
\text { ber in } \\
\text { table } 3\end{array}$} & \multicolumn{3}{|c|}{$\begin{array}{l}\text { Composition (weight } \\
\text { percent) }\end{array}$} & \multicolumn{4}{|c|}{$\begin{array}{l}\text { Phases identified ( }(=\text { large amount; } s=\text { small amount; } \\
\mathrm{m}=\text { moderate amount; } \mathrm{ss}=\text { solid solution })\end{array}$} \\
\hline & & $\mathrm{BaO}$ & $\mathrm{B}_{2} \mathrm{O}_{3}$ & $\mathrm{SiO}_{2}$ & $3 \mathrm{BaO} \cdot 3 \mathrm{~B}_{2} \mathrm{O}_{3} \cdot 2 \mathrm{SiO}_{2}$ & $\mathrm{BaO} \cdot \mathrm{B}_{2} \mathrm{O}_{3}$ & $2 \mathrm{BaO} \cdot 3 \mathrm{SiO}_{2}$ & $\mathrm{BaO} \cdot 2 \mathrm{SiO}_{2}$ \\
\hline $\begin{array}{l}a \\
b \\
c \\
d \\
e \\
f \\
g\end{array}$ & \begin{tabular}{r}
177 \\
172 \\
\hdashline 27 \\
17 \\
\end{tabular} & $\begin{array}{l}\% \\
64.8 \\
61.3 \\
65.3 \\
58.3 \\
61.5 \\
58.8 \\
67.4\end{array}$ & $\begin{array}{l}\% \\
21.4 \\
19.5 \\
19.0 \\
20.1 \\
18.1 \\
17.3 \\
20.2\end{array}$ & $\begin{array}{l}\% \\
13.8 \\
19.2 \\
15.7 \\
21.6 \\
20.4 \\
23.9 \\
12.4\end{array}$ & $\begin{array}{c}\mathrm{m} \\
\mathrm{l} \\
\mathrm{s} \\
\mathrm{l} \\
\mathrm{l} \\
\mathrm{m}\end{array}$ & $\begin{array}{c}\mathrm{l} \\
\mathrm{s} \\
\mathrm{m} \\
\mathrm{s} \\
\mathrm{l}\end{array}$ & $\begin{array}{c}\mathrm{m}(\mathrm{ss}) \\
\mathrm{s}(\mathrm{ss}) \\
\mathrm{s}(\mathrm{ss}) \\
\mathrm{s}(\mathrm{ss})\end{array}$ & $\mathrm{s}(\mathrm{ss})$ \\
\hline
\end{tabular}


c. $\mathrm{BaO} \cdot \mathrm{SiO}_{2}$

Compositions determining the barium silicate primary phase area (fig. 6) are listed in table 3, part C. Optical properties of the $\mathrm{BaO} \cdot \mathrm{SiO}_{2}$ crystals agreed well with the recorded values $[15,16,20]$. The lowest temperature for any liquidus in the field is $875 \pm 5^{\circ} \mathrm{C}$, at the ternary eutectic $\mathrm{C}$. Barium silicate and barium borate form a true binary system, with a eutectic at about $940^{\circ} \mathrm{C}$ and 43 percent, by weight, $\mathrm{BaO} \cdot \mathrm{SiO}_{2}$ (fig. $8, \mathrm{D}$ ).

\section{d. $2 \mathrm{BaO} \cdot \mathrm{SiO}_{2}$ and $\mathrm{BaO}$}

Information on the field of dibarium silicate could be obtained from only seven compositions (table 3 , part D). Six of these compositions were located along the $2 \mathrm{BaO} \cdot \mathrm{SiO}_{2}-\mathrm{BaO} \cdot \mathrm{SiO}_{2}$ boundary. The seventh (No. 70), on about the 79 percent $\mathrm{BaO}$ isopleth, formed quench growths and barium platinates to the extent that the liquidus value could not be determined; and such was the case for all compositions in the field richer in $\mathrm{BaO}$. Dashed isotherms were drawn by extrapolation from known boundary curves.

For similar reasons, together with the high temperatures involved, no liquidus values could be obtained in the $\mathrm{BaO}$ primary phase region. Because of the reaction of $\mathrm{BaO}$ at elevated temperatures with the platinum of a thermocouple, differential thermal analysis cannot be applied, as was discovered in the study of the $\mathrm{BaO}-\mathrm{B}_{2} \mathrm{O}_{3}$ binary system [4]. It is apparent, however, that the $\mathrm{BaO}$ primary field must be limited to a small area in the corner of the diagram.

\section{e. $3 \mathrm{BaO} \cdot \mathrm{B}_{2} \mathrm{O}_{3}$}

Liquidus determinations in the tribarium borate field presented most of the same difficulties as in the $2 \mathrm{BaO} \cdot \mathrm{SiO}_{2}$ and $\mathrm{BaO}$ areas; however, the three compositions studied (table 3, part E) served to delineate the tribarium borate field, which is the smallest in the system. Composition 71 (liquidus $908^{\circ} \mathrm{C}$ ) was close to the ternary eutectic C (fig. 6), and a sample quenched from $875^{\circ} \mathrm{C}$ showed three crystalline phases $\left(3 \mathrm{BaO} \cdot \mathrm{B}_{2} \mathrm{O}_{3}, \mathrm{BaO} \cdot \mathrm{B}_{2} \mathrm{O}_{3}\right.$, and $\left.\mathrm{BaO} \cdot \mathrm{SiO}_{2}\right)$ in equilibrium with small amount of glass. A ternary reaction point is postulated at $\boldsymbol{J}$ (figs. 6 and 7 ), where the crystalline phases $3 \mathrm{BaO} \cdot \mathrm{B}_{2} \mathrm{O}_{3}, \quad \mathrm{BaO}$ and $2 \mathrm{BaO} \cdot 2 \mathrm{iO}_{2}$ would be in equilibrium with glass. The invariant point $J$ might be expected to be near in both temperature and composition to the binary eutectic between $3 \mathrm{BaO} \cdot \mathrm{B}_{2} \mathrm{O}_{3}$ and $\mathrm{BaO}$ at $1,370^{\circ} \mathrm{C}$.

\section{f. $\mathrm{BaO} \cdot \mathrm{B}_{2} \mathrm{O}_{3}$}

Eighteen compositions (table 3, F) were studied in the primary phase field of barium borate, and no experimental difficulties or unusual features were encountered. Four ternary invariant points C, D, $\mathrm{H}$, and $\mathrm{B}$ (figs. 6 and 7 ) ranging in temperature from $875^{\circ}$ to $925^{\circ} \mathrm{C}$ were located on its boundaries. Barium borate forms true binary systems with both $\mathrm{BaO} \cdot \mathrm{SiO}_{2}$ and $3 \mathrm{BaO} \cdot 3 \mathrm{~B}_{2} \mathrm{O}_{3} \cdot 2 \cdot \mathrm{SiO}_{2}$ (fig. 8, D and $\mathrm{E})$. g. $\mathrm{BaO} \cdot 2 \mathrm{~B}_{2} \mathrm{O}_{3}$

The 12 compositions studied in the barium diborate field are given in table $3, \mathrm{G}$. The compound forms a true binary system with $3 \mathrm{BaO} \cdot 3 \mathrm{~B}_{2} \mathrm{O}_{3} \cdot 2 \mathrm{SiO}_{2}$ (fig. $8, \mathrm{~F}$ ) and accordingly a temperature maximum is shown on the boundary curve BF of figure 6 .

\section{h. $\mathrm{BaO} \cdot 4 \mathrm{~B}_{2} \mathrm{O}_{3}$}

Compositions for this field are listed in table $3, \mathrm{H}$. Barium tetraborate occupies a primary phase region second in extent only to that of silica. It is interesting to note, also, that less than 5 percent of the field lies in a one-liquid region, whereas more than 95 percent is in the two-liquid immiscible area (fig. 6). As the percentage of silica increased, determinations of liquidus temperatures became successively more difficult because of sluggish crystallization of the viscous melts. The boundary between the twoliquid and the one-liquid region adjacent to the $\mathrm{B}_{2} \mathrm{O}_{3}-\mathrm{SiO}_{2}$ binary was not determined and is shown as a dashed line (figs. 6 and 7 ). That this boundary must lie within several percent of the $\mathrm{B}_{2} \mathrm{O}_{3}-\mathrm{SiO}_{2}$ binary is deduced from the limited data on the indices of refraction of the low index conjugate glass (see " $n$ of quenched glass", table $3, \mathrm{H}$ ). 'The values are approximately the same or less than those for pure $\mathrm{SiO}_{2}$ or $\mathrm{B}_{2} \mathrm{O}_{3}$, both of which are about 1.46 [24].

$$
\text { i. } \mathrm{B}_{2} \mathrm{O}_{3}
$$

If the compatability triangle formed by $\mathrm{BaO} \cdot 4 \mathrm{~B}_{2} \mathrm{O}_{3}$, $\mathrm{B}_{2} \mathrm{O}_{3}$, and $\mathrm{SiO}_{2}$ (fig. 7) is correct, no additional compounds showing a stable primary phase area can be formed within its boundaries. It is then necessary to postulate an invariant point $\mathrm{M}$ located in the narrow one-liquid region adjacent to the $\mathrm{B}_{2} \mathrm{O}_{3}-\mathrm{SiO}_{2}$ binary, where the three primary phases coexist in equilibrium. In this event boric oxide would possess a very narrow primary field extending from the invariant point to pure boric oxide. Because of experimental difficulties, no attempt was made to substantiate this conclusion.

$$
\text { j. } \mathrm{SiO}_{2}
$$

The silica primary phase area (fig. 6) occupies approximately 40 percent of the phase diagram, and the silica liquidus surface is distorted by the occurrence of liquid immiscibility. The field consists of a large portion in the two-liquid area limited on three sides by a portion in the one-liquid area. A narrow strip of one-liquid area is present along the $\mathrm{B}_{2} \mathrm{O}_{3}-\mathrm{SiO}_{2}$ binary and extends to the postulated eutectic at M. Another portion of the one-liquid area extends along the $\mathrm{BaO}-\mathrm{SiO}_{2}$ binary to the eutectic at $1,374^{\circ} \mathrm{C}$ between $\mathrm{BaO} \cdot 2 \mathrm{SiO}_{2}$ and $\mathrm{SiO}_{2}$, and then penetrates the interior of the diagram adjacent to the primary phase fields of $\mathrm{BaO} \cdot 2 \mathrm{SiO}_{2}$, $3 \mathrm{BaO} \cdot 3 \mathrm{~B}_{2} \mathrm{O}_{3} \cdot 2 \mathrm{SiO}_{2}$, and $\mathrm{BaO} \cdot 2 \mathrm{~B}_{2} \mathrm{O}_{3}$. The invariant point $A$ representing the equilibrium between $\mathrm{SiO}_{2}$, $\mathrm{BaO} \cdot 2 \mathrm{~B}_{2} \mathrm{O}_{3}$ and $\mathrm{BaO} \cdot 4 \mathrm{~B}_{2} \mathrm{O}_{3}$ is at $810^{\circ} \mathrm{C}$. That the silica field must extend to the $\mathrm{BaO} \cdot 4 \mathrm{~B}_{2} \mathrm{O}_{3}$ field was 
first evident from the fact that $\mathrm{SiO}_{2}$ and $\mathrm{BaO} \cdot 4 \mathrm{~B}_{2} \mathrm{O}_{3}$ were the only primary phases identified in the twoliquid area, and fields entering a two-liquid area must persist until liquid miscibility is again encountered.

Systematic investigation of the silica field was hampered by the extremely high viscosities of all the melts and the attendant slow rate of crystal growth and approach to equilibrium. Actual liquidus values could not be obtained for a number of compositions $(115,117,119,122,123,128$ table 3, I); but phase identification in these cases was useful in approximating the limits of liquid immiscibility. In areas adjacent to the primary phase boundaries, it was not usually possible to determine the exact temperature of appearance or disappearance of the silica phase. Consequently, the silica area of the diagram is less accurate than the rest both as regards to temperature and boundaries.

Tridymite was the only form of silica identified. Even at temperatures as low as $823^{\circ} \mathrm{C}$ (No. 125 of table $3, \mathrm{I})$, or about $44 \mathrm{deg}$ below the inversion temperature of pure tridymite to quartz, no quartz was formed in any of the mixtures. In the study of the system $\mathrm{K}_{2} \mathrm{O}-\mathrm{MgO}-\mathrm{SiO}_{2}$ [25], Roedder also found tridymite persisting metastably, to a eutectic point at $715 \pm 10^{\circ} \mathrm{C}$. He concluded that a quartz liquidus stable at a higher temperature presumably existed, but because of the viscous nature of the glass, excessive time would be required to demonstrate the point.

\subsection{Stability Field of the Ternary Compound $3 \mathrm{BaO} \cdot 3 \mathrm{~B}_{2} \mathrm{O}_{3} \cdot 2 \mathrm{SiO}_{2}$}

This was the only ternary compound found in the system, and 37 compositions within its primary field were studied (table $3, J$ ). The field occupies a large portion of the glass-forming region of the system, and it was observed qualitatively that rates of devitrification (or of crystal growth) with the exception of compositions in the silica field were much slower than for compositions in the other primary phase areas.

The compound $3 \mathrm{BaO} \cdot 3 \mathrm{~B}_{2} \mathrm{O}_{3} \cdot 2 \mathrm{SiO}_{2}$ melts congruently at $1,009^{\circ} \mathrm{C}$. As seen from the spacing of the isotherms (fig. 6), its primary field is extremely flat, indicative of a high degree of dissociation of the compound in the liquid state [26]. The compound crystallizes as irregular, elongated, and fiber-like grains with positive sign of elongation. It is biaxial negative: $\alpha=1.669, \quad \beta=1.696, \quad \gamma=1.702 \quad\left(25^{\circ} \mathrm{C}\right)$, with a moderate optic axial angle of about $35^{\circ}$. The refractive index of the glass is 1.633 . When the compound is formed by devitrifying glass, polysynthetic twinning is prominent. Data from the $\mathrm{X}$-ray diffraction powder patterns are given in table 5 .

Five ternary invariant points $(\mathrm{B}, \mathrm{H}, \mathrm{G}, \mathrm{E}, \mathrm{F}$, fig. 6) are found along the boundaries of the $3 \mathrm{BaO}$. $3 \mathrm{~B}_{2} \mathrm{O}_{3} \cdot 2 \mathrm{SiO}_{2}$ field. The lowest of these invariant points is at $825^{\circ} \pm 10^{\circ} \mathrm{C}$ (point $\mathrm{F}$ ). The ternary compound forms true binary systems with $\mathrm{BaO} \cdot \mathrm{B}_{2} \mathrm{O}_{3}$
TABLE 5. X-ray diffraction data for the compound $3 \mathrm{BaO} \cdot 3 \mathrm{~B}_{2} \mathrm{O}_{3} \cdot 2 \mathrm{SiO}_{2}$, showing interplanar spacings $(d)$ and their relative intensities $(I)$

\begin{tabular}{|c|c|c|c|}
\hline$d$ & $I$ & $d$ & $I$ \\
\hline$A$ & $\%$ & $A$ & $\%$ \\
\hline 4. 96 & 35 & 2.17 & 23 \\
\hline 4. 91 & 47 & 2. 14 & 20 \\
\hline 4. 65 & 48 & 2. 09 & 25 \\
\hline 4. 09 & 17 & 2.06 & 35 \\
\hline 3.95 & 46 & 2.04 & 32 \\
\hline 3. 69 & 53 & 1. 989 & 17 \\
\hline 3.59 & 16 & 1. 898 & 11 \\
\hline 3.51 & 6 & 1. 808 & 3 \\
\hline 3. 43 & 13 & 1. 776 & 7 \\
\hline 3. 29 & $89(3)$ & 1. 759 & 10 \\
\hline 3. 09 & $100(1)$ & 1. 742 & 13 \\
\hline 2. 96 & $98(2)$ & 1. 722 & 4 \\
\hline 2.82 & 58 & 1. 659 & 6 \\
\hline 2. 75 & 12 & 1. 642 & 8 \\
\hline 2. 72 & 16 & 1. 602 & 17 \\
\hline 2. 65 & 25 & 1.579 & 4 \\
\hline 2. 50 & 50 & 1. 529 & 8 \\
\hline 2. 47 & 8 & 1. 484 & 9 \\
\hline 2.34 & 13 & 1. 461 & 4 \\
\hline 2. 20 & 23 & & \\
\hline
\end{tabular}

and with $\mathrm{BaO} \cdot 2 \mathrm{~B}_{2} \mathrm{O}_{3}$ (fig. 8, E, F). As discussed earlier, it is not believed that $3 \mathrm{BaO} \cdot 3 \mathrm{~B}_{2} \mathrm{O}_{3} \cdot 2 \mathrm{SiO}_{2}$ forms a true binary system with $\mathrm{BaO} \cdot 2 \mathrm{SiO}_{2}$, because the nature of the solid solution area is not known (fig. 8, A).

\subsection{Two-Liquid Area}

The approximate shape of the two-liquid area at the liquidus is shown in figure 6 . An interesting feature of the immiscibility area is the marked increase in concentration of $\mathrm{BaO}$ in the less siliceous liquids, proceeding toward the interior of the diagram from the baria-boric oxide binary. The maximum content of $\mathrm{BaO}$ in the ternary liquids is about 43 percent, which is an increase of 11 percent over the $\mathrm{BaO}$ content of the corresponding binary liquid. A similar observation was made in the lime-boric oxide-silica system $[3,21]$ and in the $\mathrm{ZnO}-\mathrm{B}_{2} \mathrm{O}_{3}-\mathrm{SiO}_{2}$ system [27]; but in these systems both the $\mathrm{CaO}-\mathrm{SiO}_{2}$ and the $\mathrm{CaO}-\mathrm{B}_{2} \mathrm{O}_{3}$ binaries and the $\mathrm{ZnO}-\mathrm{SiO}_{2}$ and $\mathrm{ZnO}-\mathrm{B}_{2} \mathrm{O}_{3}$ binaries, respectively, exhibit large immiscible regions. In the present system only the $\mathrm{BaO}-\mathrm{B}_{2} \mathrm{O}_{3}$ binary possesses a liquid immiscibility region, but the flat liquidus portion of the $\mathrm{BaO}-\mathrm{SiO}_{2}$ system indicating an approach to two-liquid immiscibility as discussed earlier (fig. 5) is probably a factor contributing to the wide extent of liquid immiscibility in the ternary system. In a study of the effect of lanthanum and barium in glass-forming systems, Brewster and his coworkers [1] present some very limited data on immiscibility in the system $\mathrm{BaO}-\mathrm{B}_{2} \mathrm{O}_{3}-\mathrm{La}_{2} \mathrm{O}_{3}$. Their data indicate that in this system, the addition of $\mathrm{La}_{2} \mathrm{O}_{3}$ to the binary system $\mathrm{BaO}-\mathrm{B}_{2} \mathrm{O}_{3}$ causes a decrease in extent of liquid immiscibility.

Geller and Bunting [28] in an investigation of the system $\mathrm{PbO}-\mathrm{B}_{2} \mathrm{O}_{3}-\mathrm{SiO}_{2}$ found a large region of twoliquid immiscibility similar to that in the $\mathrm{CaO}-\mathrm{B}_{2} \mathrm{O}_{3}$ $\mathrm{SiO}_{2}$ system. The $\mathrm{PbO}-\mathrm{B}_{2} \mathrm{O}_{3}$ binary has an immiscibility gap [29], but none has been reported in the $\mathrm{PbO}-\mathrm{SiO}_{2}$ binary $[30,31]$. The immiscibility area, nevertheless, shows an increase in concentration of 
TABLE 6. Tie-line compositions in the immiscibility area

\begin{tabular}{|c|c|c|c|c|c|c|c|c|c|c|c|}
\hline \multirow{3}{*}{$\begin{array}{l}\text { Compo- } \\
\text { sition } \\
\text { number }\end{array}$} & \multicolumn{9}{|c|}{ Compositions } & \multirow{3}{*}{ Liquidus } & \multirow{3}{*}{ Separation } \\
\hline & \multicolumn{3}{|c|}{ Initial a } & \multicolumn{3}{|c|}{$\mathrm{High} \mathrm{SiO}_{2}$ glass } & \multicolumn{3}{|c|}{ Low $\mathrm{SiO}_{2}$ glass } & & \\
\hline & $\mathrm{BaO}$ & $\mathrm{B}_{2} \mathrm{O}_{3}$ & $\mathrm{SiO}_{2}$ & $\mathrm{BaO}$ & $\mathrm{B}_{2} \mathrm{O}_{3}$ & $\mathrm{SiO}_{2}$ & $\mathrm{BaO}$ & $\mathrm{B}_{2} \mathrm{O}_{3}$ & $\mathrm{SiO}_{2}$ & & \\
\hline $\begin{array}{l}104 \\
118\end{array}$ & $\begin{array}{c}\% \\
18.0 \\
20.0\end{array}$ & $\begin{array}{c}\% \\
62.0 \\
40.0\end{array}$ & $\begin{array}{c}\% \\
20.0 \\
40.0\end{array}$ & $\left\{\begin{array}{c}\% \\
29.62 \\
29.15 \\
32.55\end{array}\right.$ & $\begin{array}{c}\% \\
64.21 \\
64.88 \\
42.79\end{array}$ & $\begin{array}{c}\% \\
6.17 \\
5.97 \\
24.66\end{array}$ & $\begin{array}{r}\% \\
4.80 \\
11.84 \\
3.27\end{array}$ & $\begin{array}{c}\% \\
59.24 \\
60.02 \\
33.83\end{array}$ & $\begin{array}{c}\% \\
35.96 \\
28.14 \\
62.90\end{array}$ & \} $\begin{array}{c}{ }^{\circ} \mathrm{C} \\
\quad 872 \\
>823\end{array}$ & $\left\{\begin{array}{l}925^{\circ} \mathrm{C} / 16 \mathrm{hr} . \\
1,250^{\circ} \mathrm{C} / 1 \mathrm{hr} . \\
1,250^{\circ} \mathrm{C} / 1 \mathrm{hr} .\end{array}\right.$ \\
\hline
\end{tabular}

s Formulated from end members and not analyzed.

$\mathrm{PbO}$ in the less siliceous liquids proceeding toward the interior of the diagram from the $\mathrm{PbO}-\mathrm{B}_{2} \mathrm{O}_{3}$ binary The $\mathrm{PbO}$ concentration varies from about 45 percent on the $\mathrm{PbO}-\mathrm{B}_{2} \mathrm{O}_{3}$ binary, to a maximum of about 60 percent. Inspection of the $\mathrm{PbO}-\mathrm{SiO}_{2}$ diagram showed that the liquidus curve had not been determined beyond about 35 percent, by weight, of $\mathrm{SiO}_{2}$. It is postulated, therefore, from the shape of the ternary immiscibility boundary that the liquidus curve in the unexplored area of the $\mathrm{PbO}-\mathrm{SiO}_{2}$ binary might be S-shaped as in the $\mathrm{BaO}-\mathrm{SiO}_{2}$ system.

A study was made of the compositions of conjugate liquids formed within the immiscible area by melting of two compositions, one in the $\mathrm{BaO} \cdot 4 \mathrm{~B}_{2} \mathrm{O}_{3}$ and the other in the $\mathrm{SiO}_{2}$ primary field. The results are listed in table 6 and shown in a portion of figure 7. In figure 7 , the solid dots in the $\mathrm{BaO} \cdot 4 \mathrm{~B}_{2} \mathrm{O}_{3}$ and $\mathrm{SiO}_{2}$ fields represent the two initial compositions; and the open circles connected by straight lines, the analyzed values of the separated compositions.

The initial compositions were prepared from the end members, barium carbonate, silica gel and boric acid, by the described method of repeated grinding and partial fusion. These two compositions were not analyzed at the start, but the two separated compositions, regardless of the degree of separation, and the associated initial one must lie on a straight lind provided there has been no change on the initial composition during the heat treatment. Figure 7 shows that the three associated compositions do lie essentially on a straight line; and it is reasonable to assume that composition number 118 (in the silica field) was altered slightly during its preparation. In no case was separation of the conjugate immiscible liquids complete, but the intersection of the line passing through them with the boundaries of the immiscible area is a tie line for the particular temperature of separation.

The quintuple line KL is the boundary separating the $\mathrm{BaO} \cdot 4 \mathrm{~B}_{2} \mathrm{O}_{3}$ and $\mathrm{SiO}_{2}$ primary fields. Any point on the quintuple line represents the equilibrium between two liquids of compositions $\mathrm{K}$ and $\mathrm{L}$, two solids $\mathrm{BaO} \cdot 4 \mathrm{~B}_{2} \mathrm{O}_{3}$ and $\mathrm{SiO}_{2}$, and vapor.

As separation of the immiscible liquids could not be achieved by gravity at the liquidus temperature or slightly above, the actual separations were made well above the liquidus. For composition number 104 (fig. 7 and table 6) separation was tried at two temperatures, $925^{\circ}$ and $1,250^{\circ} \mathrm{C}$, in an attempt to obtain information on the effect of temperature on the extent of two-liquid immiscibility. Because of incomplete separation, it was not possible to draw any conclusions from the lengths of the two tie lines. Inspection of the data, however, in two other respects was revealing: (1) The tie lines for the two temperatures of separation for composition 104 (fig. 7) showed a small but significant angle between them. This rotation of the tie line with increasing temperature is positive indication that the compositions of the conjugate liquids are changing with temperature [32]. (2) The indices of refraction, measured with the polarizing microscope, of clear fragments of the low silica (high index) conjugate glasses were as follows: $1.542 \pm 0.002$ and $1.540 \pm 0.002\left(25^{\circ} \mathrm{C}\right)$ for the glasses separated as liquids at $925^{\circ}$ and $1,250^{\circ}$ $\mathrm{C}$, respectively. Extrapolations from figure 10 showing the indices of refraction of the quenched glasses indicate that a decrease in index of refraction of 0.002 corresponds to a decrease in $\mathrm{BaO}$ content of about 2 percent. Measurements of the high silica conjugate glasses were more difficult and less exact, but the index of refraction of the glass separated at $1,250^{\circ} \mathrm{C}$ was definitely higher (between 1.45 and 1.46) than the glass separated at $925^{\circ} \mathrm{C}$ (just under 1.45). It seems, therefore, from these data that the two-liquid area decreases in extent with increasing temperature.

A few preliminary experiments were performed using the high-temperature centrifuge developed by T. F. Newkirk and Fred Ordway ${ }^{4}[33]$. Much better separations than by gravity were obtained, and this approach offers new promise in the study of liquid immiscibility. Their equipment, however, is suitable only for small samples, and micromethods of analysis would have to be applied.

Recent studies on several selected compositions located near the boundary of the $\mathrm{BaO}$ rich immiscible liquid have shown that it is possible to quench these compositions as two glasses at one temperature and as one glass at some higher temperature, presumably where complete miscibility is encountered. Work is in progress with the view of determining the shape of the immiscibility dome.

\subsection{Insofract diagram for quenched glasses}

Figure 10 shows an isofract diagram for the quenched glasses. It can be seen that barium oxide

${ }^{4}$ Associated with the Portland Cement Association Fellowship at the Nationa Bureau of Standards. 


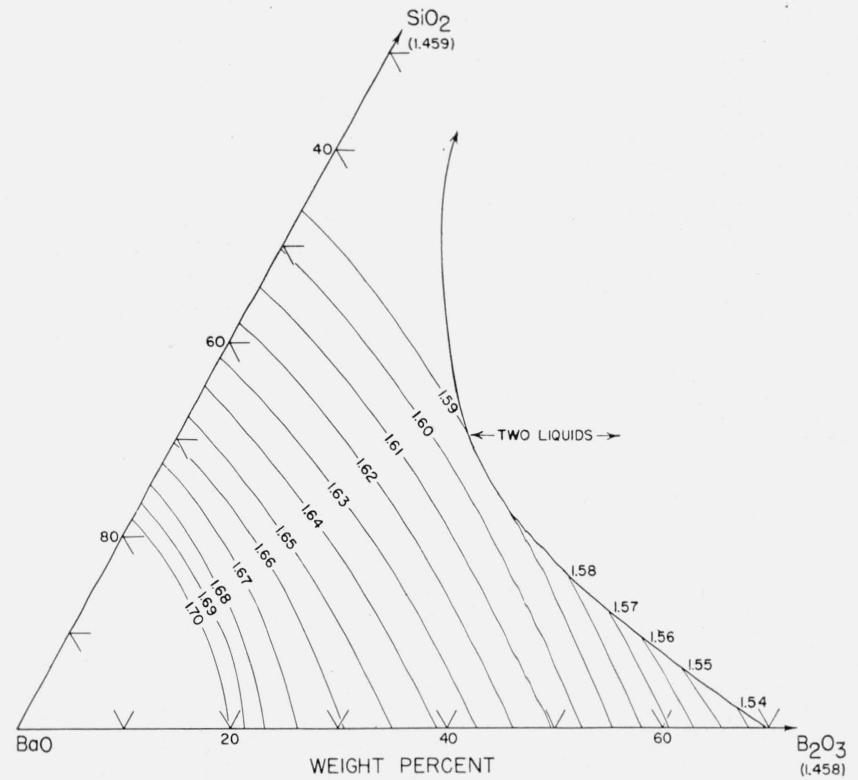

FIGURE 10. Indices of refraction of quenched glasses in the system $\mathrm{BaO}-\mathrm{B}_{2} \mathrm{O}_{3}-\mathrm{SiO}_{2}$.

imparts the greatest change in refractive index and that the increase in refractive index for a given addition of $\mathrm{BaO}$ becomes greater as the $\mathrm{BaO}$ corner is approached. Of particular interest is the convex nature of the isofracts, with the $\mathrm{BaO}$ vertex as a common center of curvature. Thus, indices of glasses lying on a $\mathrm{BaO}$ isopleth will show a maximum at about the center of the isopleth. These findings agree with those of Hamilton and his coworkers [2], who have made a detailed and accurate study of indices and densities of annealed glasses in the system. Their index values are higher, in general, by a small but irregular amount from those of the present study, in which no attempt was made to relieve the internal stresses and strains caused by quenching.

\section{Summary}

The equilibrium diagram for the system $\mathrm{BaO}$ $\mathrm{B}_{2} \mathrm{O}_{3}-\mathrm{SiO}_{2}$ was constructed from data, obtained essentially by the quenching method, on 178 ternary compositions. All the binary compounds showed primary fields in the ternary system. One new compound, $3 \mathrm{BaO} \cdot 3 \mathrm{~B}_{2} \mathrm{O}_{3} \cdot 2 \mathrm{SiO}_{2}$, melting at $1,009^{\circ} \mathrm{C}$ and possessing an extremely flat primary field was found, and its optical and X-ray properties described. Table 7 lists the compositions and liquidus temperatures of the ternary invariant points together with the crystalline phases in equilibrium with liquid.

The $\mathrm{BaO} \cdot 4 \mathrm{~B}_{2} \mathrm{O}_{3}$ and $\mathrm{SiO}_{2}$ primary phase areas include almost 70 percent of the diagram, and over these areas exists a large region of two-liquid immiscibility. On the basis of rotation of a tie line in the immiscibility region at two temperatures and of a change in index of refraction of the conjugate glasses at the two temperatures, it was concluded that the immiscibility gap decreases with increasing temperature.

Applying the method of eutectic matrix compound composition showed that $2 \mathrm{BaO} \cdot 3 \mathrm{SiO}_{2}$ is probably a compound and not merely a particular composition, even though the exact nature and extent of the $2 \mathrm{BaO} \cdot 3 \mathrm{SiO}_{2}$ solid-solution field could not be ascertained. The solid-solution region between $2 \mathrm{BaO}$. $3 \mathrm{SiO}_{2}$ and $\mathrm{BaO} \cdot 2 \mathrm{SiO}_{2}$ extends into the interior of the diagram and was found to be complex.

An isofract diagram for the quenched glasses shows that barium oxide exerts the greatest influence on increase of refractive index. The isofracts lie nearly parallel to the borosilicate binary.

A self-adjusting, bridge-type controller suitable for precise temperature control of a quenching furnace was described briefly.

TABLE 7. Ternary invariant points in the system $\mathrm{BaO}-\mathrm{B}_{2} \mathrm{O}_{3}-\mathrm{SiO}_{2}$

\begin{tabular}{|c|c|c|c|c|c|c|c|}
\hline \multirow[b]{2}{*}{ Point a } & \multirow[b]{2}{*}{ Phases in equilibrium with one liquid } & \multirow[b]{2}{*}{ Type } & \multicolumn{4}{|c|}{ Composition $\mathrm{b}$} & \multirow[b]{2}{*}{$\begin{array}{l}\text { Temper- } \\
\text { ature }\end{array}$} \\
\hline & & & $\mathrm{BaO}$ & $\mathrm{B}_{2} \mathrm{O}_{3}$ & $\mathrm{SiO}_{2}$ & $\begin{array}{l}\text { Approx- } \\
\text { imate } \\
\text { limits }\end{array}$ & \\
\hline $\begin{array}{l}\text { A } \\
\text { B } \\
\text { C } \\
\text { D } \\
\text { E }-\cdots\end{array}$ & $\begin{array}{l}\mathrm{BaO} \cdot 2 \mathrm{~B}_{2} \mathrm{O}_{3}, \mathrm{BaO} \cdot 4 \mathrm{~B}_{2} \mathrm{O}_{3}, \mathrm{SiO}_{2} \\
\mathrm{BaO} \cdot \mathrm{B}_{2} \mathrm{O}_{3}, \mathrm{BaO} \cdot 2 \mathrm{~B}_{2} \mathrm{O}_{3}, 3 \mathrm{BaO} \cdot 3 \mathrm{~B}_{2} \mathrm{O}_{3} \cdot 2 \mathrm{SiO}_{2} \\
3 \mathrm{BaO} \cdot \mathrm{B}_{2} \mathrm{O}_{3}, \mathrm{BaO} \cdot \mathrm{B}_{2} \mathrm{O}_{3}, \mathrm{BaO} \cdot \mathrm{SiO}_{2} \\
\mathrm{BaO} \cdot \mathrm{B}_{2} \mathrm{O}_{3}, \mathrm{BaO} \cdot \mathrm{SiO}_{2}, 2 \mathrm{BaO} \cdot 3 \mathrm{SiO}_{2} \\
3 \mathrm{BaO} \cdot 3 \mathrm{~B}_{2} \mathrm{O}_{3} \cdot 2 \mathrm{SiO}_{2}, \mathrm{BaO} \cdot 2 \mathrm{SiO}_{2}, \mathrm{SiO}_{2}\end{array}$ & \begin{tabular}{l} 
Eutectic \\
do \\
\hdashline do \\
do do
\end{tabular} & $\begin{array}{l}\% \\
38.7 \\
54.2 \\
77.4 \\
68.0 \\
51.8\end{array}$ & $\begin{array}{l}\% \\
53.8 \\
44.0 \\
19.0 \\
18.6 \\
21.2\end{array}$ & $\begin{array}{r}\% \\
7.5 \\
1.8 \\
3.6 \\
13.4 \\
27.0\end{array}$ & $\begin{array}{r}\% \\
\pm 0.3 \\
\pm .3 \\
\pm .3 \\
\pm .3 \\
\pm 1.0\end{array}$ & $\begin{array}{l}{ }^{\circ} \mathrm{C} \\
810 \pm 10 \\
875 \pm 5 \\
875 \pm 5 \\
920 \pm 10 \\
950 \pm 20\end{array}$ \\
\hline $\begin{array}{l}\mathrm{F} \\
\mathrm{G} \\
\mathrm{H} \\
\mathrm{I} \\
\mathrm{J}\end{array}$ & $\begin{array}{l}3 \mathrm{BaO} \cdot 3 \mathrm{~B}_{2} \mathrm{O}_{3} \cdot 2 \mathrm{SiO}_{2}, \mathrm{BaO} \cdot 2 \mathrm{~B}_{2} \mathrm{O}_{3}, \mathrm{SiO}_{2} \\
3 \mathrm{BaO} \cdot 3 \mathrm{~B}_{2} \mathrm{O}_{3} \cdot 2 \mathrm{SiO}_{2}, \mathrm{BaO} \cdot 2 \mathrm{SiO}_{2}, 2 \mathrm{BaO}_{2} \cdot 3 \mathrm{SiO}_{2} \\
\mathrm{BaO} \cdot \mathrm{B}_{2} \mathrm{O}_{3}, \mathrm{BaO} \cdot \mathrm{SiO}_{2}, 2 \mathrm{BaO} \cdot 3 \mathrm{SiO}_{2} \ldots \\
3 \mathrm{BaO} \cdot \mathrm{B}_{2} \mathrm{O}_{3}, \mathrm{BaO} \cdot \mathrm{SiO} 2,2 \mathrm{BaO} \cdot \mathrm{SiO}_{2} \\
\mathrm{BaO}, 3 \mathrm{BaO} \cdot \mathrm{B}_{2} \mathrm{O}_{3}, 2 \mathrm{BaO} \cdot \mathrm{SiO}_{2}\end{array}$ & 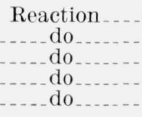 & $\begin{array}{l}43.4 \\
60.2 \\
67.5 \\
78.3 \\
87\end{array}$ & $\begin{array}{l}45.4 . \\
18.4 \\
18.5 \\
17.3 \\
12\end{array}$ & $\begin{array}{c}11.2 \\
21.4 \\
14.0 \\
4.4 \\
1\end{array}$ & $\begin{array}{l} \pm 1.0 \\
\pm .5 \\
\pm .3 \\
\pm .6 \\
\mathrm{c} ?\end{array}$ & $\begin{array}{l}825 \pm 10 \\
980 \pm 5 \\
925 \pm 10 \\
962 \pm 10 \\
<1,370\end{array}$ \\
\hline \multirow[t]{2}{*}{ Md $d . .}$. & $\mathrm{BaO} \cdot 4 \mathrm{~B}_{2} \mathrm{O}_{3}, \mathrm{~B}_{2} \mathrm{O}_{3}, \mathrm{SiO}_{2}$ & \multirow[t]{2}{*}{ Eutectic } & \multirow[t]{2}{*}{$<2$} & \multirow[t]{2}{*}{$>30$} & \multirow[t]{2}{*}{$<30$} & \multirow[t]{2}{*}{ c? } & \multirow[t]{2}{*}{$<450$} \\
\hline & Phases in equilibrium with two liquids $(\mathrm{K}, \mathrm{L}$ ) & & & & & & \\
\hline $\begin{array}{l}\mathrm{K} \ldots . . \\
\mathrm{L} \ldots\end{array}$ & $\begin{array}{l}\mathrm{BaO} \cdot 4 \mathrm{~B}_{2} \mathrm{O}_{3}, \mathrm{SiO}_{2} \\
\mathrm{BaO} \cdot 4 \mathrm{~B}_{2} \mathrm{O}_{3}, \mathrm{SiO}_{2}\end{array}$ & $\begin{array}{l}\text { (e) } \\
\text { (e) }\end{array}$ & $\begin{array}{c}37.4 \\
1\end{array}$ & $\begin{array}{l}54.6 \\
40\end{array}$ & $\begin{array}{c}8.0 \\
59\end{array}$ & $\underset{c}{ \pm 1.0}$ & $815 \pm 10$ \\
\hline
\end{tabular}

a Figures 6 and 7 .

b Determined by extrapolation of established boundary curves to intersection; values given in weight percent. c Because exact location of one or more boundaries not determined. d Postulated [21]

e Quintuple line joins compositions of liquids $\mathrm{K}$ and $\mathrm{L}$. 
The authors take pleasure in expressing their appreciation to Floyd A. Mauer for the design and construction of the self-adjusting bridge-type furnace controller; to Edgar H. Hamilton, G. W. Cleek, and O. H. Grauer, of the Glass Section of the National Bureau of Standards, for preparation of the seven devitrified glass samples used in determining the nature of the $2 \mathrm{BaO} \cdot 3 \mathrm{SiO}_{2}$ composition; and to Barbara M. Sullivan for obtaining the X-ray diffraction powder pattern data.

\section{References}

[1] Gordon F. Brewster, Norbert J. Kreidl, and Tvler G. Pett, Lanthanum and barium in glass-forming systems, J. Soc. Glass Tech. 31, 153 (1947).

[2] Edgar H. Hamilton, G. W. Cleek, and O. H. Grauer, Some properties of $\mathrm{BaO}-\mathrm{B}_{2} \mathrm{O}_{3}-\mathrm{SiO}_{2}$ glasses and a theory of glass structure. (Publication pending.)

[3] E. P. Flint and Lansing S. Wells, The system limeboric oxide-silica, J. Research NBS 17, 727 (1936) RP941.

[4] Ernest M. Levin and Howard F. MeMurdie, The system $\mathrm{BaO}-\mathrm{B}_{2} \mathrm{O}_{3}$, J. Research NBS 42, 131 (1949) RP1956.

[5] E. S. Shepherd, G. A. Rankin, and F. E. Wright, Binary systems of alumina with silica, lime, and magnesia, Äm. J. Sci. [4] 28, 293 (1909).

[6] W. L. Hill, G. T. Faust, and D. S. Reynolds, The binary system $\mathrm{P}_{2} \mathrm{O}_{5}-2 \mathrm{CaO} \cdot \mathrm{P}_{2} \mathrm{O}_{5}$, Am. J. Sci. 242, 457 (1944)

[7] H. S. Roberts, The Geophysical Laboratory furnace thermostat, J. Opt. Soc. Am. 11, 171 (1925)

[8] Henry Shenker, John L. Lauritzen, Jr., and Robert J. Corruccini, Reference tables for thermocouples, NBS Cir. 508 (1951)

[9] R. J. Corruccini, Differences between the international temperature scales of 1948 and 1927, J. Research NBS 43, 133 (1949) RP2014.

[10] B. Hague, A. C. bridge methods, 5th ed. (Sir Isaac Pitman \& Sons, Ltd, London, 1946).

[11] G. W. Morey, The ternary system $\mathrm{Na}_{2} \mathrm{O}-\mathrm{B}_{2} \mathrm{O}_{3}-\mathrm{SiO}_{2}, \mathrm{~J}$. Soc. Glass Tech. 35, 270 (1951).

[12] J. W. Greig, Immiscibility in silica melts, Am. J. Sci. [5], 13, 1-44, 133-54 (1927).

[13] A. Cousen and W. E. S. Turner, Studies of the glasses boric oxide-silica, J. Soc. Glass Tech. 12, 169-190 (1928)

[14] N. K. Dertev and T. H. Khudyakova, Solubility of silica in $\mathrm{SiO}_{2}-\mathrm{B}_{2} \mathrm{O}_{3}$ glasses (Zhadnov Ind. Inst., Gorki) J. Applied Chem. USSR 23, 839-45 (1950).

[15] P. Eskola, The silicates of strontium and barium, Am. J. Sci. [5], 4, 345 (1922).
[16] R. H. Thomas, Phase equilibrium in a portion of the ternary system $\mathrm{BaO}-\mathrm{Al}_{2} \mathrm{O}_{3}-\mathrm{SiO}_{2}, \mathrm{~J}$. Am. Ceram. Soc. 33, [2], 36 (1950).

[17] Ya. I. Ol'shanskir, Equilibrium of two immiscible liquids in silicate systems of alkali-earth metals, Doklady Akad. Nauk., SSSR, 76 [1], 93 (1951); J. Am. Ceram. Soc. absts., 34 [8], 145 (1951)

[18] W. Eitel, Silicate melt equilibria, Par. 67 (Rutgers University Press, New Brunswick, N. J. 1951).

[19] R. F. Geller, A resistor furnace with some preliminary results up to $2,000^{\circ} \mathrm{C}$, J. Research NBS $\mathbf{2 7}, 555$ (1941) RP1443; R. F. Geller, P. J. Yavorsky, B. L. Steierman, and A. S. Creamer, Studies of binary and ternary combinations of magnesia, calcia, baria, beryllia, alumina, thoria and zirconia in relation to their use as porcelains, J. Research NBS 36, 277 (1946) RP1703.

[20] Alfred E. Austin, X-ray diffraction data for compounds in systems $\mathrm{Li}_{2} \mathrm{O}-\mathrm{SiO}_{2}$ and $\mathrm{BaO}-\mathrm{SiO}_{2}, \mathrm{~J}$. Am. Ceram. Soc. 30 [7], 218 (1947).

[21] G. W. Morey and Earl Ingerson, System $\mathrm{CaO}-\mathrm{B}_{2} \mathrm{O}_{3}$ $\mathrm{SiO}_{2}, \mathrm{Am}$. Mineralogist 22 [1], 38 (1937).

[22] Wilfrid R. Foster, Contribution to the interpretation of phase diagrams of ceramists, J. Am. Ceram. Soc. 34 [5], 151 (1951).

[23] Wilfrid R. Foster, A study of solid state reaction in the ternary system $\mathrm{MgO}-\mathrm{ZrO}_{2}-\mathrm{SiO}_{2}, \mathrm{~J}$. Am. Ceram. Soc. 34 [10], 302 (1951).

[24] A. N. Winchell, The microscopic characters of artificial inorganic solid substances or artificial minerals, 2nd ed. (John Wiley \& Sons, Inc., New York, N. Y., 1931.)

[25] Edwin Woods Roedder, The system $\mathrm{K}_{2} \mathrm{O}-\mathrm{MgO}-\mathrm{SiO}_{2}$, Am. J. Sci. 249, 81-130 and 224-248 (1951).

[26] Alexander Findlay, The phase rule and its applications, p 113 (Dover Publications, New York, N. Y., 1945).

[27] Earl Ingerson, G. W. Morey and O. F. Tuttle, The systems $\mathrm{K}_{2} \mathrm{O}-\mathrm{ZnO}-\mathrm{SiO}_{2}, \quad \mathrm{ZnO}-\mathrm{B}_{2} \mathrm{O}_{3}-\mathrm{SiO}_{2}, \quad \mathrm{Zn}_{2} \mathrm{SiO}_{4}-$ $\mathrm{Zn}_{2} \mathrm{GeO}_{4}$, Am. J. Sci. 246, 31 (1948).

[28] R. F. Geller and E. N. Bunting, The system $\mathrm{PbO}-\mathrm{B}_{2} \mathrm{O}_{3}$ $\mathrm{SiO}_{2}$, J. Research NBS 23, 275 (1939) RP1231.

[29] R. F. Geller and E. N. Bunting, The system $\mathrm{PbO}-\mathrm{B}_{2} \mathrm{O}_{3}$, J. Research NBS 18, 585 (1937) RP995.

[30] R. F. Geller, A. S. Creamer, and E. N. Bunting, The system: $\mathrm{PbO}-\mathrm{SiO}_{2}, \mathrm{~J}$. Research NBS 13, 237 (1934) RP705.

[31] F. P. Hall and Herbert Insley, Phase diagrams for ceramists, J. Am. Ceram. Soc., Part II (Nov. 1947).

[32] G. Masing, Ternary systems, p. 103 (Reinhold Publishing Corp., New York, N. Y., 1944).

[33] T. F. Newkirk and Fred Ordway, The high-temperature centrifuge and its use in phase equilibrium studies, Bull. Am. Ceram. Soc. 31 [3] (abst., 54th Annual Meeting Program 1952).

Washington, May 12, 1953 